Article

\title{
Genomic fabric remodeling in metastatic clear cell renal cell carcinoma (ccRCC): A new paradigm and proposal for a personalized gene therapy approach
}

\author{
Dumitru A Iacobas ${ }^{1, *}$, Victoria E Mgbemena ${ }^{2}$, Sanda Iacobas ${ }^{3}$, Kareena M Menezes ${ }^{4}$, Huichen \\ Wang ${ }^{4}$ and Premkumar B Saganti $4,5^{*}$ \\ 1 Personalized Genomics Laboratory, CRI Center for Computational Systems Biology, Roy G Perry College \\ of Engineering, Prairie View A\&M University, Prairie View, TX 77446; daiacobas@pvamu.edu \\ 2 Department of Biology, MD and S Brailsford College of Arts and Sciences, Prairie View A\&M University, \\ Prairie View, TX 77446; vemgbemena@pvamu.edu \\ 3 Department of Pathology, New York Medical College, Valhalla, NY 10595, U.S.A. sandaiacobas@gmail.com \\ ${ }^{4}$ CRI Radiation Institute for Science \& Engineering, MD and S Brailsford College of Arts and Sciences, \\ Prairie View A\&M University, Prairie View, TX 77446; kmmenezes@pvamu.edu; huwang@pvamu.edu; \\ 5 Department of Physics, MD and S Brailsford College of Arts and Sciences, Prairie View A\&M University, \\ Prairie View, TX 77446; pbsaganti@pvamu.edu \\ * Correspondence: daiacobas@pvamu.edu, pbsaganti@pvamu.edu; Tel: +1 (936) 2619626
}

Simple Summary: Clear cell renal cell carcinoma (ccRCC) accounts for $\sim 70 \%$ of all kidney cancers. However, despite decades of research, the molecular mechanisms responsible for the disease and, more importantly, the therapeutic solution are still works in progress. We present a 3D transcriptomic analysis of the chest wall metastasis (MET), two primary cancer nodules (PTA, PTB) and normal surrounding tissue in the right kidney surgically removed from a ccRCC patient. The studies revealed that even histopathological equally classified (PTA and PTB) cancer nodules from the same kidney have different transcriptomic topologies. Hence the need for a custom made therapeutic solution, tailored for each patient as soon as cancer is detected. Using the Gene Master Regulator (GMR) approach we identified TASOR, FAM27C and ALG13 as the most legitimate gene targets for PTA, PTB and MET, and propose molecular mechanisms by which expression manipulation of TASOR, and ALG13 may selectively impact the cancer cells.

\begin{abstract}
Published transcriptomic data from surgically removed metastatic clear cell renal cell carcinoma (ccRCC) samples were re-analyzed from the Genomic Fabric Perspective that considers the transcriptome a multi-dimensional mathematical object, constrained by a dynamic set of expression correlations among genes. Every gene in the chest wall metastasis (MET), two primary tumors (PTA, PTB) and the surrounding normal tissue (NOR) of the right kidney was characterized by three independent measures: average expression level (AVE), relative expression variation (REV) and expression correlation (COR) with each other gene. AVE was used to determine the regulation of the genomic fabrics of cCRCC, apoptosis, chemokine and VEGF signaling pathways. REV quantified the alteration of the transcripts' abundances control, while COR determined the remodeling of the transcriptomic networks of chemokine signaling and oxidative phosphorylation genes. The gene hierarchy was established in based on Gene Commanding Height and the Gene Master Regulators (GMR) TASOR (PTA), FAM27C (PTB) and ALG13 (MET) and DAPK3 (NOR) were identified in each profiled region. We predict that TASOR overexpression would block transcription in PTA but not in PTB, while slightly stimulating it in NOR. Silencing of ALG3 would slow-down the cell-cycle in all three cancer regions with practically no effect in NOR.
\end{abstract}

Keywords: ALG13; apoptosis; cell cycle; chemokine signaling; FAM27C; genomic medicine; oxidative phosphorylation; TASOR; VEGF signaling; VHL. 


\section{Introduction}

The American Cancer Society estimates that by the end of 2020, U.S.A. will have 73,750 (45,520 men, 28,230 women) new cases of kidney (KC) and pelvis cancers, and 14,830 (9,860 men and 4,970 women) death because of these cancers [1]. Smoking (18\%), obesity (21\%), hypertension (21\%) along with family history are major contributors to the development of the disease, although about $40 \%$ of cases cannot be associated these risk factors [2]. If detected and treated early (while the cancer is confined to the kidney), the survival rate is very high ( $93 \%$ at 5 years). However, for advanced stage, when cancer has spread to lungs, brain, bones and other distant parts of the body, there is only $12 \%$ survival rate at 5 years [1]. The American Joint Committee on Cancer (AJCC) uses the TNM system to classify the four $\mathrm{KC}$ stages based on: the size and extent of the tumor $(\mathrm{T})$, spread to the nearby nodes (N) and metastasis to distant cells (M) [3]. In parallel, Fuhrman grade system [4] of KC stages uses the microscopic appearance of the haematoxylin and eosin stained cells.

Over $90 \%$ of KCs are Renal Cell Carcinoma (RCC), arising primarily in the renal parenchyma. The RC stable 2:1 male to female ratio incidence across populations suggests possible sexual dimorphism in its genetic susceptibility [5]. RCC is classified into several subtypes, out of which 75\% are clear cell RCC (ccRCC) [6]. Despite advancements in detection and treatment, diagnosing aggressive subtypes present challenges due to tumor heterogeneity, biopsy sample availability, different response rates to treatment and the non-negligible technical noise of the molecular biology assessment methods.

Numerous meta-analyses (e.g.: [7-9]) tried to identify the gene biomarkers whose altered sequence or/and expression may serve to diagnose the kidney cancer form, and whose restoration may help to cure it. Among others, the 26.0 release (September 08, 2020) of the Genomic Data Commons Data Portal [10] includes 59,612 mutations detected in 20,426 genes sequenced from 3,295 (2,110 male and 1,185 female) KC cases. As such, there is practically no gene without mutation reported in at least one kidney cancer case.

Owing to low frequency in the screened population, no mutated gene emerged as a clear indicator of KC. Thus, only VHL (von Hippel-Lindau tumor suppressor, E3 ubiquitin protein ligase; 5.64\%), PBRM1 (polybromo 1; 4.83\%), TTN (titin; 4.67\%) and MUC4 (mucin 4, cell surface associated; $2.67 \%$ ) were mutated in more than $2 \%$ of cases. However, using data from The Cancer Genome Atlas, Rickets et al. [11] concluded that in the cohort of profiled RCC patients, VHL was mutated in $41,3 \%$ of the cases and PRBM1 in 38.1\%, although neither of these high frequencies was associated with the overall survival. SETD2 (SET domain containing 2), MUC16 (mucin 16, cell surface associated), BAP1 (BRCA1 associated protein-1), DST (dystonin), KMT2C (lysine (K)-specific methyltransferase 2C), LRP2 (low density lipoprotein receptor-related protein 2), MACF1 (microtubule-actin crosslinking factor 1), PKHD1 (polycystic kidney and hepatic disease 1), CUBN (cubilin) and OBSCN (obscurin, cytoskeletal calmodulin and titin-interacting RhoGEF) are among the genes altered in more than $1 \%$ of cases. Even alterations of the most anticipated genes: TP53 (tumor protein p53; 1.09\%), MTOR (mechanistic target of rapamycin; 1.06\%) and PTEN (phosphatase and tensin homolog; 0.97\%) are not frequent enough to be considered as good indicators. Moreover, a gene can harbor several mutations; for instance, the above cited genes had between 33 (MTOR) and 199 (TTN) mutations. Not only are the mutated genes in almost all chromosomes, but the encoded proteins are involved in a wide diversity of biological processes. Therefore, it is hard to delineate a general molecular mechanism responsible for $\mathrm{KC}$. 
VHL [12,13], VEGF (vascular endothelial growth factor, [14]), CAIX (carbonic anhydrase IX, [15]), PTEN [15], CR1 (complement C3b/C4b receptor 1, [16]), MERTK (MER proto-oncogene tyrosine kinase [17]), PIK3CA (phosphatidylinositol-4,5-bisphosphate 3-kinase catalytic subunit alpha [18]) and AKT1 (AKT serine/threonine kinase 1 [19]) are among the most popular KC biomarkers. The $\mathrm{PI}$ KK/Akt pathway, one that is altered in several cancers, has been evaluated as a potential target for therapies [20].

However, together with the considered biomarker, hundreds of other genes are mutated or/and regulated in $\mathrm{KC}$, and their contributions are neglected without enough evidence that they are verifiably negligible. The "other" affected genes are in unique combinations not only from patient to patient, but also among cancer nodules in the same tumor (as proven below) and at distant time points during the progression of the disease or recovery of the same person. The collection of the affected genes is unique for each human because the gathering together of the transcriptomic conditioning factors is never perfectly repeatable. In previous papers we have shown that the transcriptome depends on the: genetic background [21], sex [22], age [23], medical history [24], hormonal activity [25], environmental conditions [26], exposure to stress [27], toxins [28], treatment [29] and external stimuli [30]. By evidence, there is no way to have exactly the same combination of factors or/and identical influences of a particular factor in distinct individuals. Moreover, even in the same tumor there are histopathologically distinct regions [31, 32], a clear indication of different gene expression profiles (distinct cell morphologies result from different expression of cytoskeleton genes and subcellular localization of the encoded proteins). In addition, out of the $\sim 20,000$ genes in the human genome there are 20 million distinct combinations of two genes and almost 2,000 billion of three genes. These numbers make impossible to assign predictive values to all sets of more than two altered genes. Though, there are authors who tested the diagnostic and therapeutic merits of some particular combinations of two (e.g. [33]) or three (e.g. [34]) gene biomarkers for RCC.

The Genomic Fabric Paradigm (GFP) [35] offers a holistic alternative to the cancer biomarkers approach by quantifying cancer-induced transcriptome alterations of the genomic fabrics associated with functional pathways. The genomic fabric of a biological process was defined as "the transcriptome associated to the most stably expressed and interconnected gene network responsible for that biological process" [35]. GFP considers the transcriptome as a multi-dimensional mathematical object constrained by a dynamic set of expression correlations among the genes. GFP assigns three independent characteristics to each quantified gene in each phenotype: average expression level, expression variability and expression coordination with each other gene [36]. GFP paved the way for the Gene Master Regulators (GMR) approach [37] that we believe provides the best targets for the cancer gene therapy [38].

\section{Results}

\subsection{Independent characteristics of the quantified genes in each profiled region}

We have analyzed previously published gene expression data from chest wall metastasis (MET), two primary tumor nodules (denoted by PTA, PTB) and the surrounding normal tissue (NOR) of the right kidney of a ccRCC patient [39]. The experimental design which used four biological replicates, provided for each quantified gene in each region, three independent characteristics: average expression level (AVE), Relative Expression Variation (REV) and expression coordination (COR) with each other gene (see Methods).

In total, we quantified 13,314 unigenes in each of the 16 profiled samples (four biological replicates per region). Thus, in addition to the 13,314 AVEs that would be considered in any 
traditional transcriptomic study, we analyzed also 13,314 REVs and $(13,314 \times 13,313 / 2=) 88,624,641$ COR values, a tremendous increase (by 6,658.5x) of the transcriptomic information. Expression level, variability and correlation of genes probed by multiple spots (such are MIEF1 (mitochondrial elongation factor 1) and SRRT (serrate, RNA effector molecule) probed by 20 spots each) were computed as indicated in the Methods section. Genes not adequately quantified in one sample were eliminated from the analysis.

Figure 1 presents the three independent characteristics in the PTA and PTB regions for the first 50 alphabetically ordered (out of 130) quantified genes involved in the KEGG-determined chemokine signaling (CS) pathway [40]. The chemokine signaling pathway was analyzed for the importance of its components in modulating cancer cells ability to grow, proliferate, invade, and metastasize (e.g. [41, 42]). Our analysis went beyond the traditional AVE by determining also how much the expression of each gene is allowed to fluctuate (by determining REV) and how its fluctuations are correlated with the fluctuations of other genes in the same region. Thus, the information for one gene is doubled by considering its REV and increased by adding the number of correlations with other quantified genes. As such, in addition to the $4 \times 13,314=53,256$ average expression levels, we analyzed also 53,256 REVs and 354,498,564 correlation coefficients (= 4 regions x 13,314 x 13,313/2 distinct gene pairs).

$V H L$, a multipurpose adaptor protein, was selected to illustrate the independence of the expression coordination with respect to the average expression level and expression variation because of the alleged role of this gene in $\mathrm{KC}[12,13,34]$. VHL is considered also a circuit breaker in the evolution of ccRCC [43]. However, correlation with any other gene should equally support the independence of the three characteristics. 


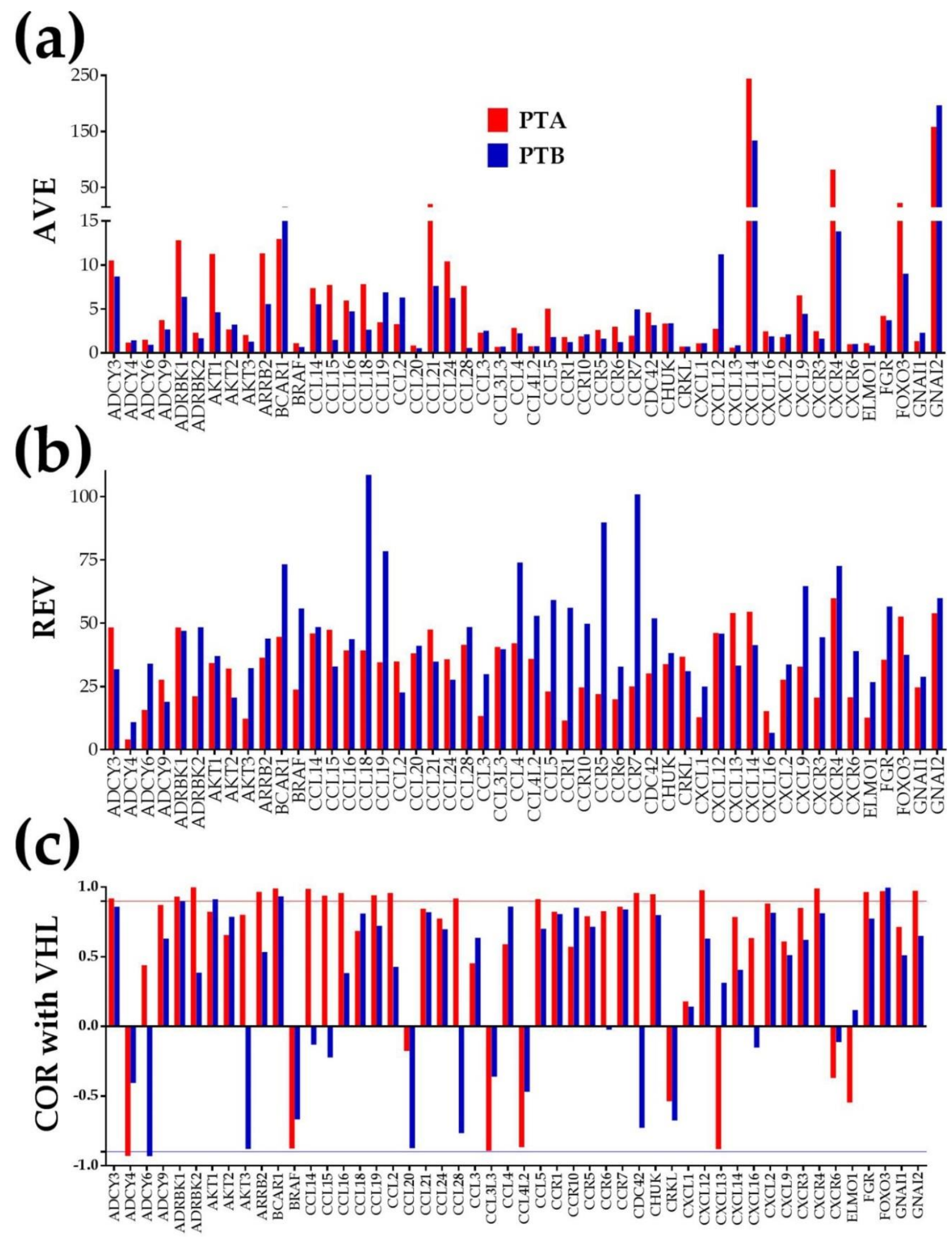

Figure 1: The three independent characteristics (AVE, REV, COR) of the first 50 alphabetically ordered chemokine signaling genes in the two profiled cancer nodules (PTA, PTB). Note the independence of the three characteristics in each nodule and the differences between the two nodules although they have the same Fuhrman grade and were isolated from the same (right) kidney. 
In addition to the evident independence of the three characteristics of these genes in each region, of note in Fig. 1 are also the differences between the two primary nodules. Thus, only for the illustrated 50 CS genes, $A D C Y 6, A K T 1 / 3, C C L 15 / 18 / 28 / 5, C C R 6, C X C L 16$ have significantly higher expression in PTA and ADCY4, CCL2, CXCL12 and GNG7 in PTB. There are also significant differences in REV. Moreover, the number of significantly coordinated chemokines with VHL was 19 $(14.62 \%)$ synergistically and $1(0.77 \%)$ antagonistically in PTA and 3 synergistically $(2.31 \%)$ and 1 antagonistically in PTB. Figure 2 illustrates the separation of the NOR, PTA, PTB and MET transcriptomes. Figure 2a presents the separation in the two adenylate cyclase genes (ADCY4$A D C Y$ 6) subspace of the expression levels. In this subspace, the center of PTA is at 0.97 expression units from the center of NOR, the center of PTB at 0.77 units and that of MET at 1.47 units. With respect to NOR, each of the two genes was up-regulated in all three cancer regions; $A D C Y 4$ (by 1.66x, $2.01 \mathrm{x}, 1,57 \mathrm{x}$ ), $A D C 6$ (by: 2.29x, 1.40x, 3.14x). However, $A D C Y 4$ had significantly higher expression in PTB than in PTA and MET, while ADCY6 had significantly lower expression in PTB.

Figure $2 \mathrm{~b}$ shows the separation in the 3D space of percent differences between the cancer nodules and NOR with respect to all three independent variables when all 130 quantified genes from the chemokine signaling pathway and their inter-coordination are considered. Differences with respect to the normal tissue (NOR, placed in the origin of the three orthogonal axes) were computed as indicated in the Methods section. In this space, the 3D (AVE-diff, REV-diff, COR-diff) coordinates of the cancer nodules are: PTA (42.6, -10.5, 103.8), PTB (13.2, -6.7, 18.9), and MET (59.0, -10.9, 118.8). Thus, PTA, PTB and MET are respectively at 112.10, 23.98 and 133.27 units from NOR. An interesting result is that while the average expression level and inter-coordination of CS genes increased in all cancer nodules, the expression variability decreased.

Remarkably, although all three cancer nodules were isolated from the same person (PTA and PTB even from the same kidney) and categorized as the same Fuhrman grade 3 metastatic ccRCC, their transcriptomic alterations with respect to the normal kidney tissue were largely different from each-other. Hence, in this subspace limited to only the quantified 130 chemokine signaling genes, the distance between PTA and PTB is 89.9, between PTA and MET is 22.36 and between PTB and MET is 110.16. The much smaller distance between MET and PTA than between MET and PTB suggest that MET cells came most likely from the PTA region

\section{(a)}

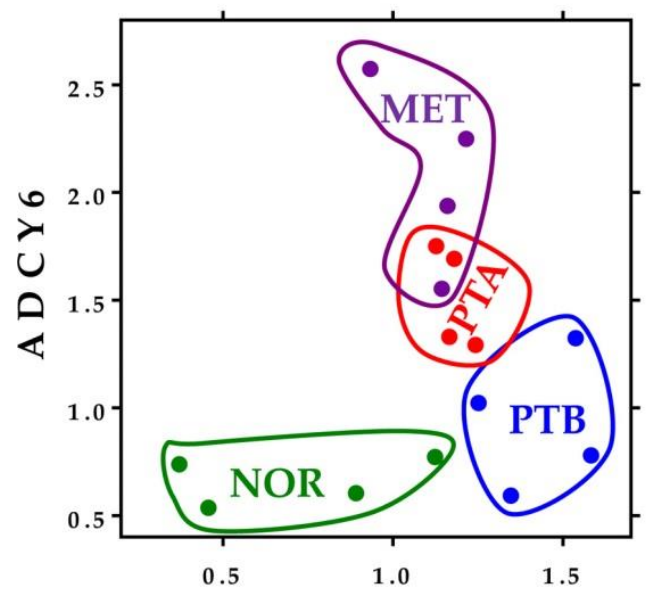

A D C Y 4

\section{(b)}

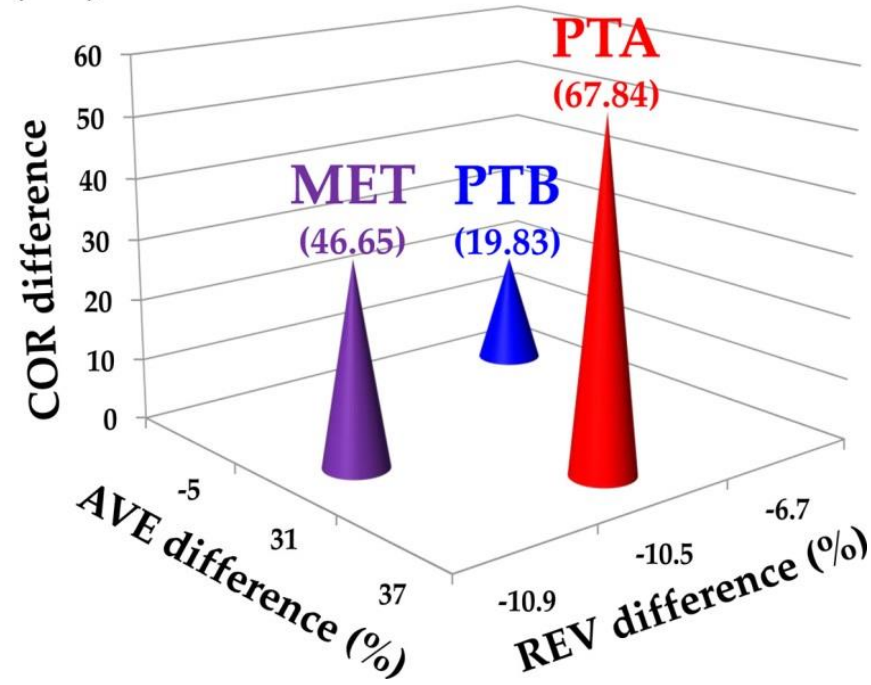

Figure 2: Separation of ccRCC nodules in: (a) two-gene expression level subspace; (b) 3D space of independent measures. (a) was limited to the gamma G proteins 7 and 11, while (b) presents the normalized scores for all 130 chemokine signaling genes. Each colored bullet in (a) represents the expressions levels of the two genes in one of the quarters of the indicated region. The tips of the cones 
in (b) represent the 3D coordinates of the alteration of the quantified genes from the chemokine signaling pathway in the three profiled cancer nodules.

\subsection{Regulation of the ccRCC functional pathway}

Figure 3 shows the regulation of the genes responsible for the RCC in each of the three cancer nodules with respect to the normal tissue. The genes and their interlinkages were selected from the KEGG-determined human renal cell carcinoma pathway [44]. As indicated by P (= p-value) computed with hypergeometric distribution of the number of regulated vs. number of quantified genes in the Gene Ontology (GO) term [45]), the ccRCC pathway was significantly regulated in all three profiled regions. However, regulation of ccRCC genes was different in the three nodules and even opposite between PTA and PTB for VEGFA (vascular endothelial growth factor A).

(a) PTA vs NOR

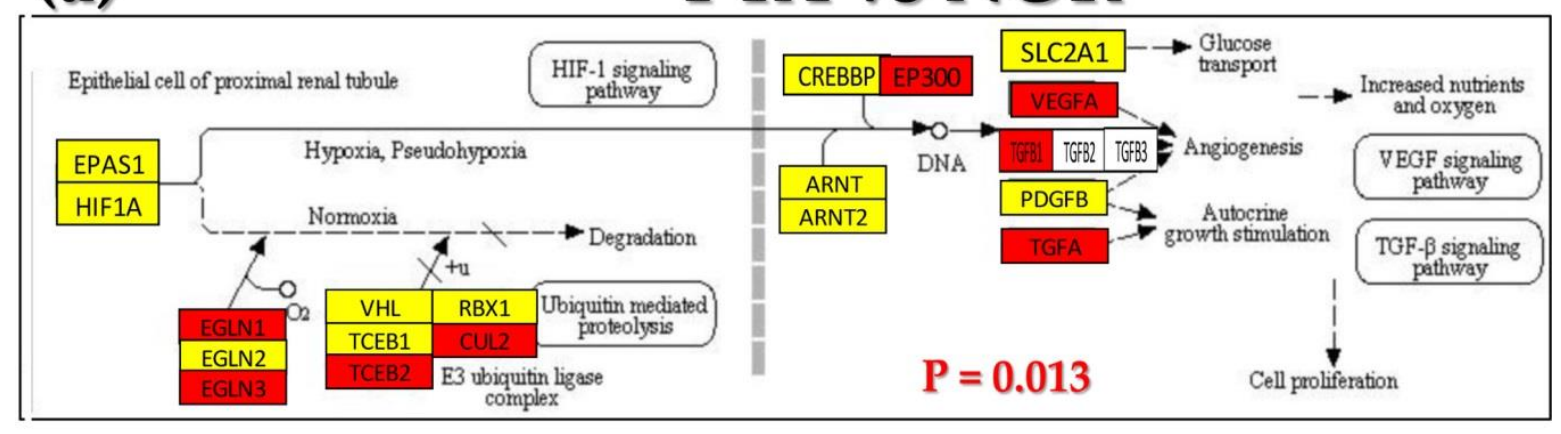

(b)

PTB vs NOR

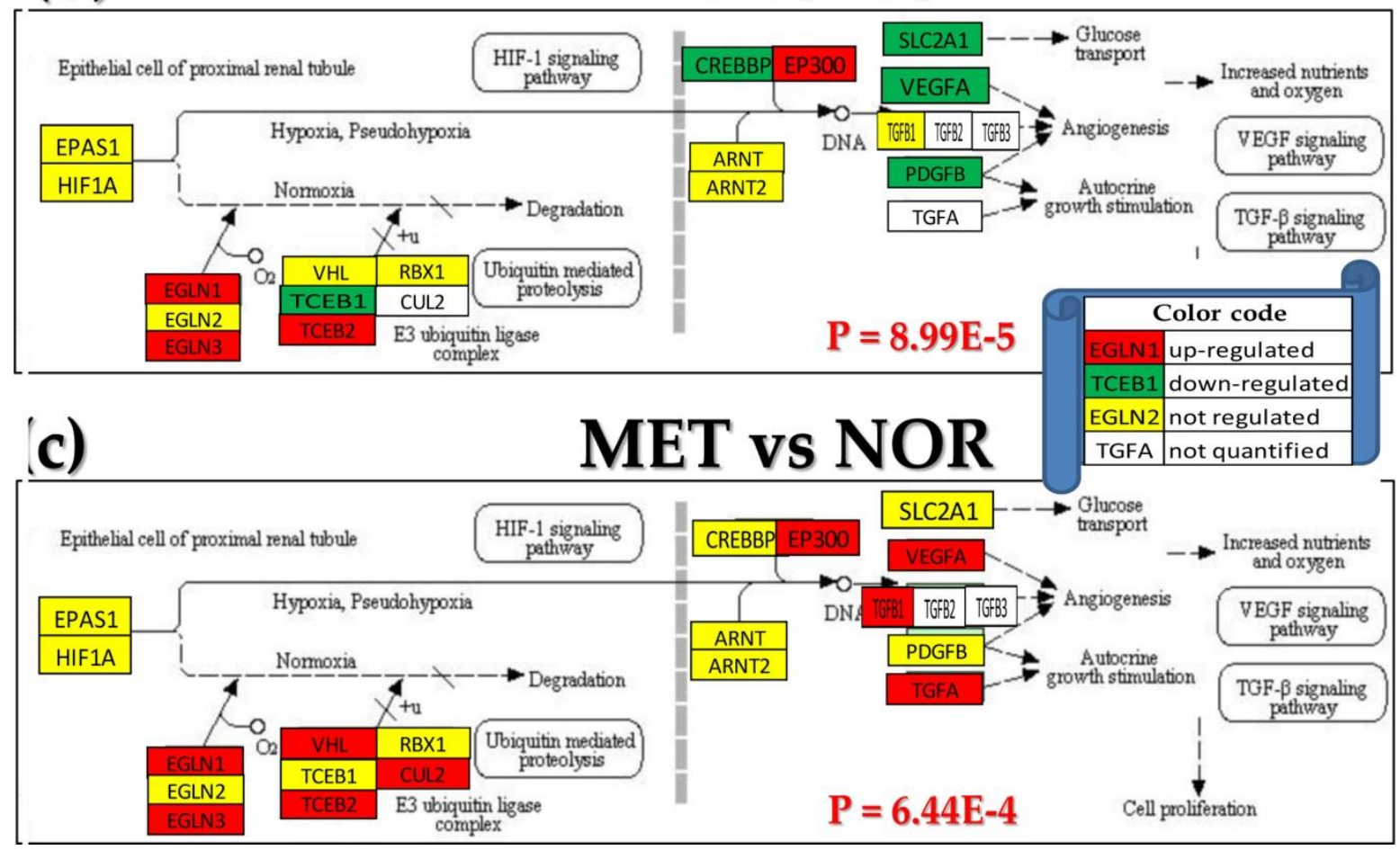

Figure 3: Regulation of the conventional KEGG-determined ccRCC pathway in the three cancer 
nodules (PTA, PTB, MET) with respect to the normal tissue (NOR) from the right kidney. Numbers are the p-values of the regulation of the pathway genes in the indicated comparison. Significantly regulated genes: CREBBP (CREB binding protein), CUL2 (cullin 2), EGLN1/3 (egl-9 family hypoxiainducible factor 1/3), EP300 (E1A binding protein p300), TCEB1/2 (transcription elongation factor B (SIII), polypeptide 1/2), TGFA/B1 (transforming growth factor alpha/beta 1), VEGFA, VHL.

\subsection{Regulation of the apoptosis pathway}

Figure 4 presents the regulation of the KEGG-determined apoptosis pathway [46] in each profiled cancer region with respect to the normal kidney tissue. Again, there are notable differences between the equally ranked cancer nodules PTA and PTB. Thus, there are genes regulated in PTA but not in PTB: AKT1/3, BCL2, IL3RA, IL3RAP, PRKA1RA, RELA, TNFRSF10A, TNFRSF1A. Other genes were regulated in PTB but not in PTA: AIFM1, DFFB, RIPK1, PIK3R2, PPP3CA, PRKA1RB, TNFRSF10B. Also, from the perspective of this pathway regulation, MET is closer to PTA than to PTB.

\section{(a)}

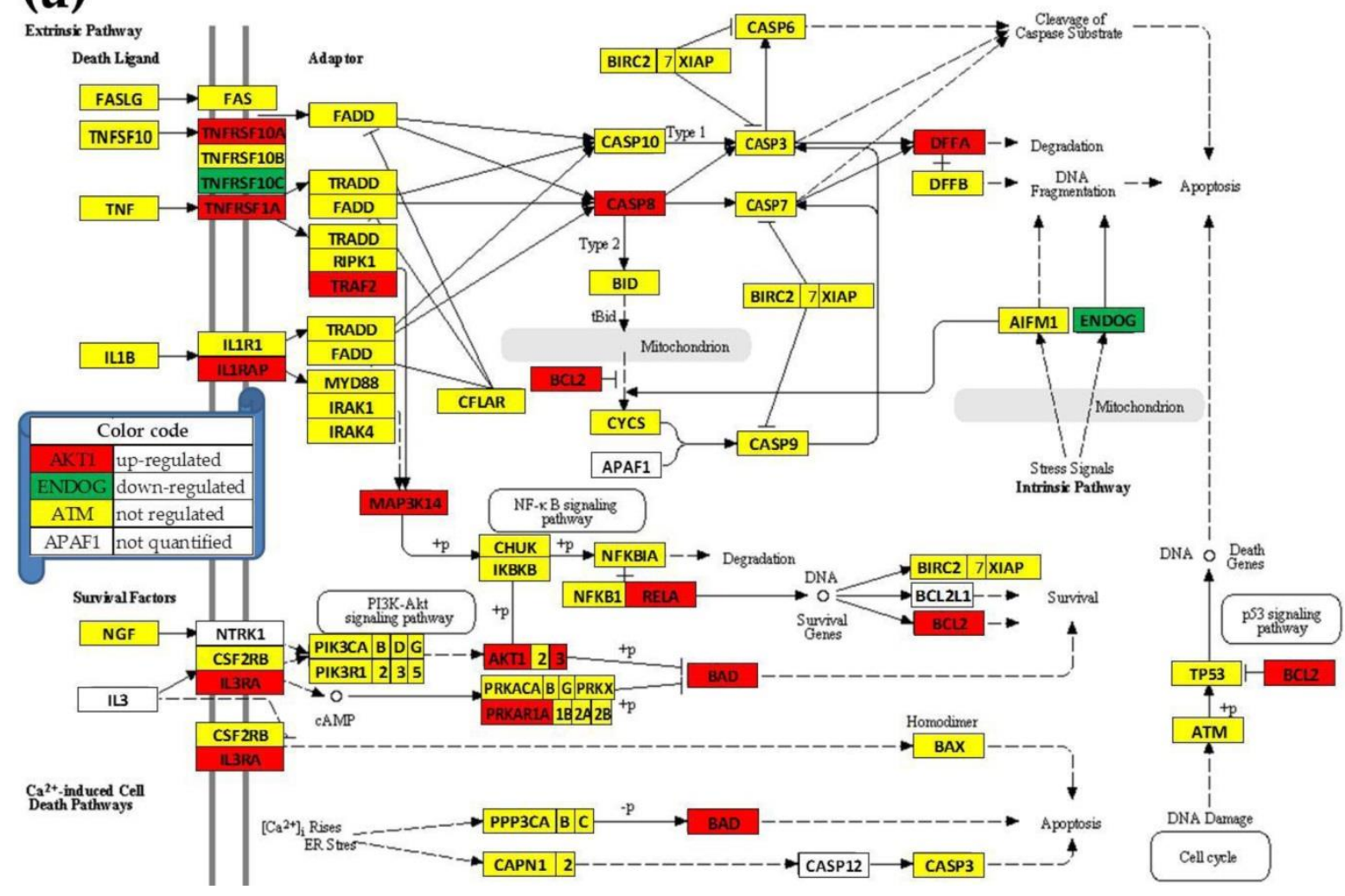


(b)

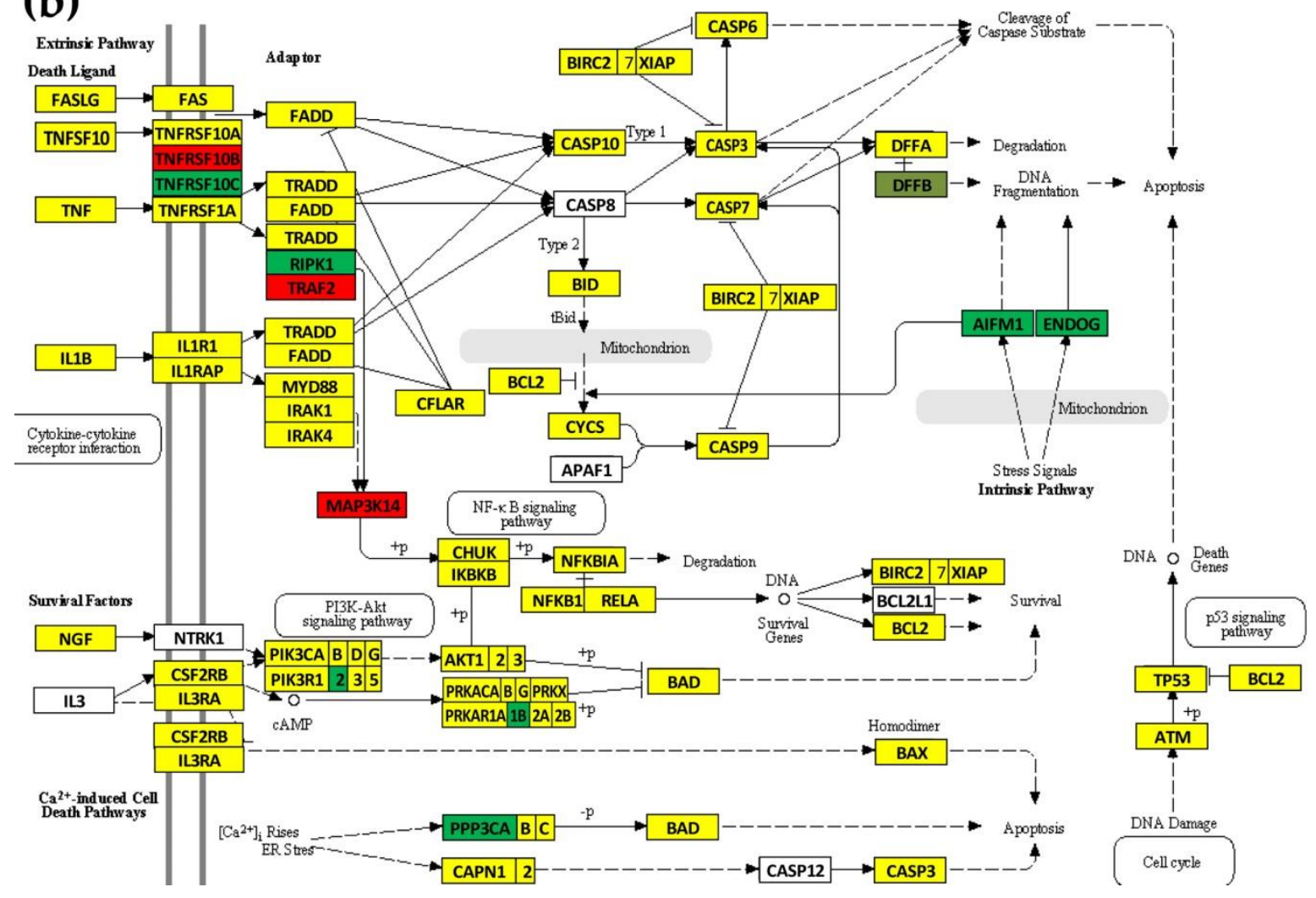

(c)

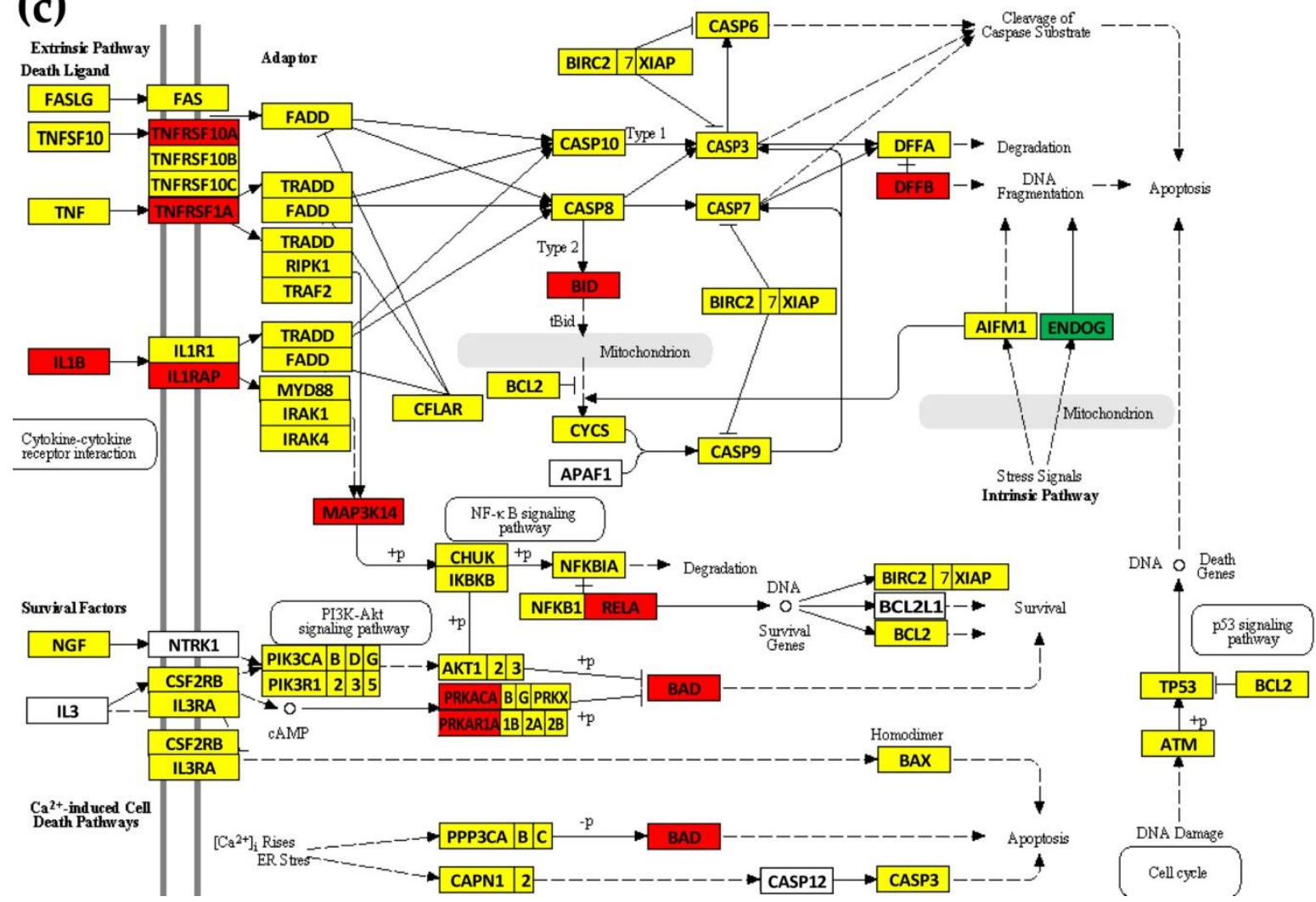

Figure 4 Regulation of the apoptosis pathway in: (a) PTA, (b) PTB and (c) MET regions with respect

to NOR. Regulated genes: AIFM1 (apoptosis-inducing factor, mitochondrion-associated, 1), AKT1/3 
(v-akt murine thymoma viral oncogene homologs), $B A D$ (BCL2-associated agonist of cell death), $B C L 2$ (B-cell CLL/lymphoma 2), BID (BH3 interacting domain death agonist (BID), DFFA/B (DNA fragmentation factors, $40 \mathrm{kDa}$, polypeptide (caspase-activated DNase), ENDOG (endonuclease G, IL1B (interleukin 1, beta), IL1RAP IL3RA (interleukin receptors), MAP3K14 (mitogen-activated protein kinase kinase kinase 14), PPP3CA (protein phosphatase 3, catalytic subunit, alpha isozyme), PRKACA (protein kinase, cAMP-dependent, catalytic, alpha), $P R K A R 1 A / B$ (protein kinases, cAMPdependent, regulatory, type I), RELA (RELA proto-oncogene, NF-kB subunit), RIPK1 (receptor interacting serine/threonine kinase 1), TNFRSF1A/10A/10B/10C (members of the tumor necrosis factor receptor superfamily), TRAF2 (TNF receptor-associated factor 2.

\subsection{Regulation of the VEGF signaling pathway}

Figure 5 presents the regulation of the genes involved in the KEGG-determined VEGF signaling pathway [47] in the three cancer regions with respect to the normal kidney tissue. This pathway was analyzed because it is one of the most important target in KC therapy [48]. Unfortunatelly, KDR (kinase insert domain receptor), the main mediator of the major growth factor (VEGF) [49] was not quantified in this experiment. However, we quantified almost all other downstream genes in all regions. Interestingly, in PTA and MET where VEGF was up-regulated there are substantially more up-regulated genes (10 and 15) than in PTB (4) where VEGF was down-regulated. While in NOR, phospholipase A2, group IVA (cytosolic, calcium-dependent) (PLA2G4A) was not expressed, in all three cancer regions it was turned on. PLA2G4A is an important target for prevention and treatment of cancers. [50]. Prostaglandin-endoperoxide synthase 2 (prostaglandin $\mathrm{G} / \mathrm{H}$ synthase and cyclooxygenase) (PTGS2), whose upregulation is associated with carcinogenesis and cancer progression [51], was also turned on in MET.

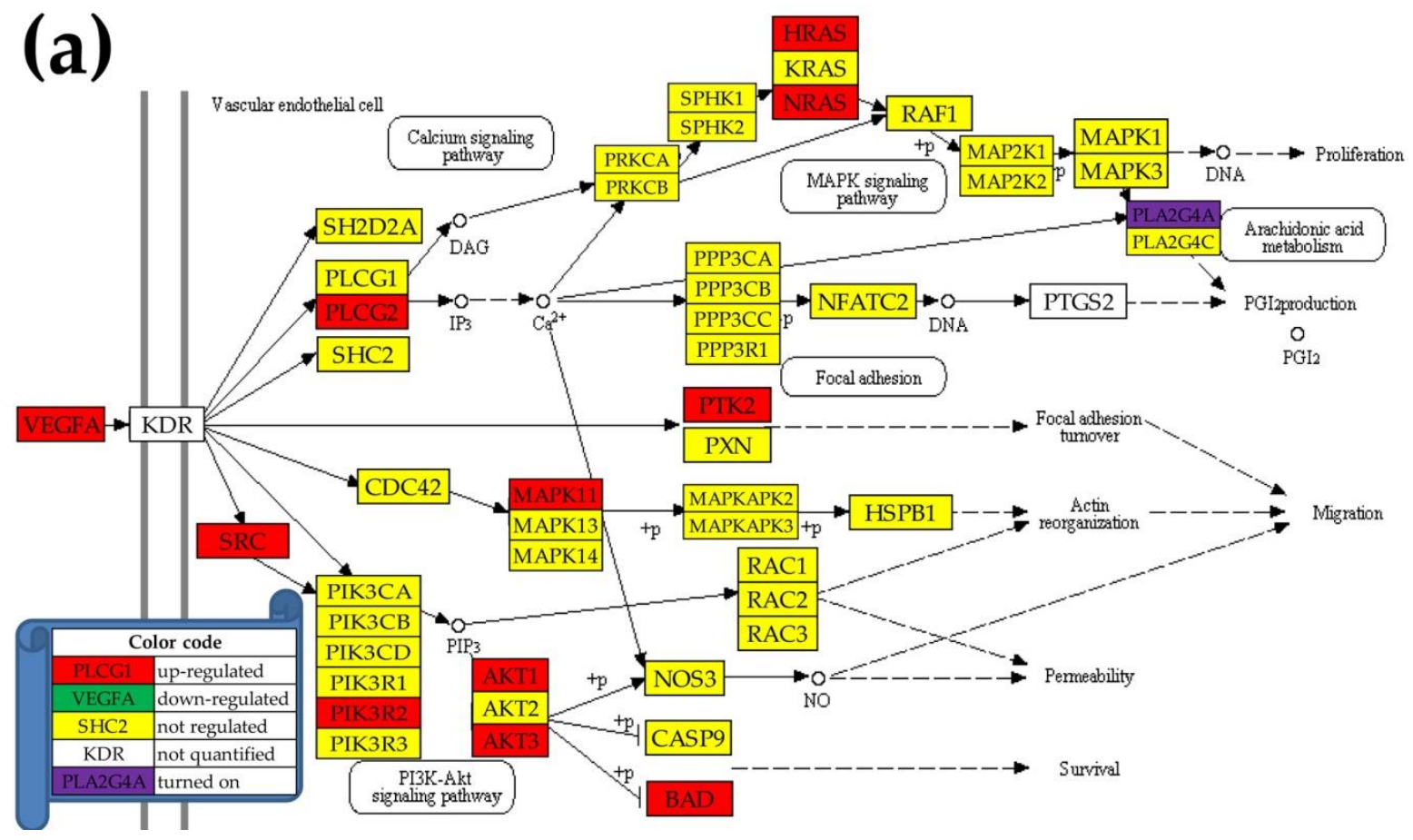



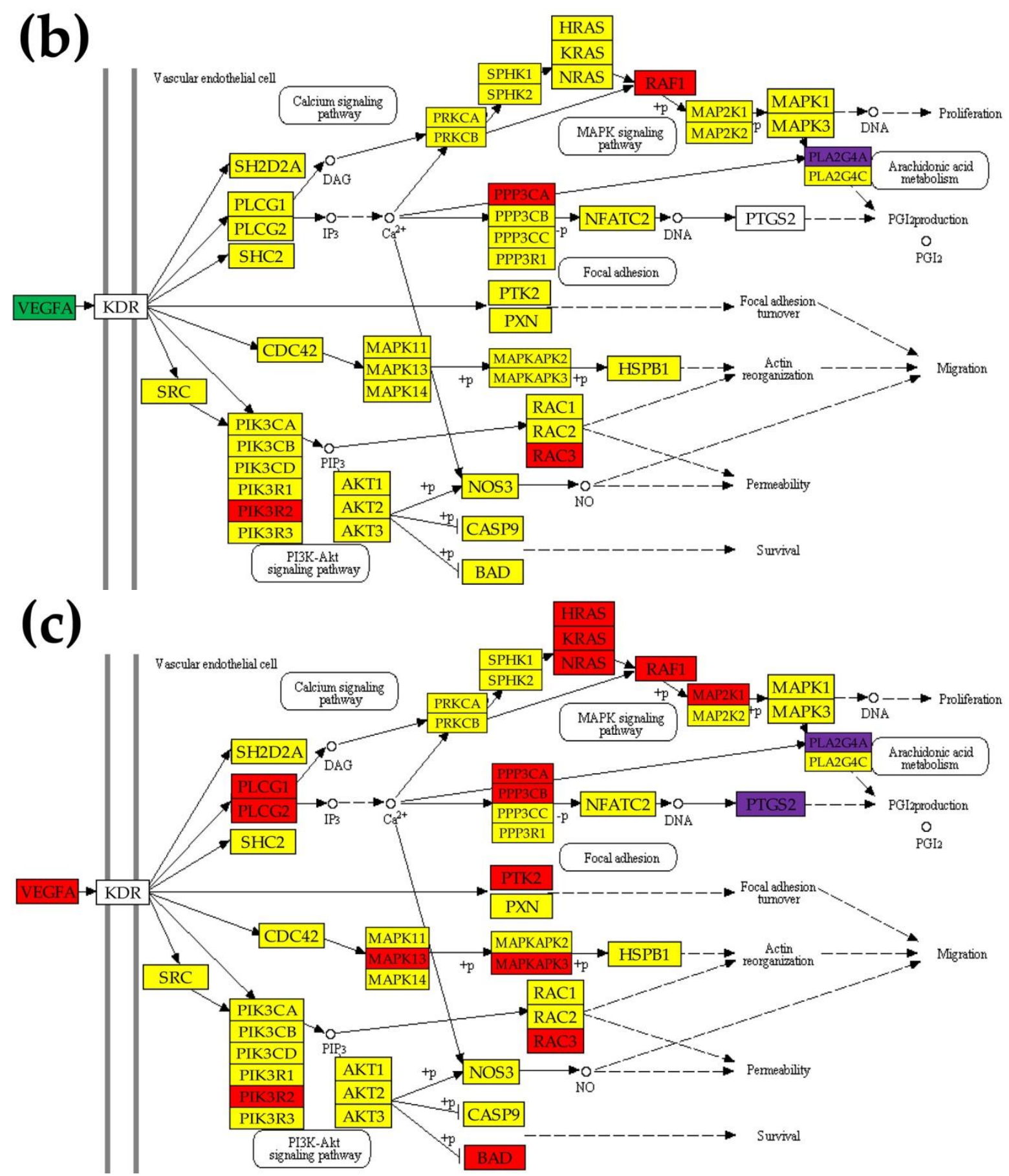

Figure 5 Regulation of the VEGF signaling pathway in (a) PTA, (b) PTB, (c) MET. Regulated genes: HRAS (Harvey rat sarcoma viral oncogene homolog), KRAS (Kirsten rat sarcoma viral oncogene homolog), MAPK11/13 (mitogen-activated protein kinases 11/13), NRAS (neuroblastoma RAS viral (v-ras) oncogene homolog), PIK3R2 (phosphoinositide-3-kinase, regulatory subunit 2 (beta)), PLCG2 (phospholipase C, gamma 2 (phosphatidylinositol-specific)), PTK2 (protein tyrosine kinase 2), SRC (v-src avian sarcoma (Schmidt-Ruppin A-2) viral oncogene homolog). 
The median REV of the 130 quantified CS unigenes in the four regions were: 41.50 (NOR), 37.12 (PTA), 38.73 (PTB) and 36.97 (MET). Hence, as illustrated in Fig. 2b, with respect to NOR, the median REV decreased in PTA by $10.5 \%$ in PTB by $6.7 \%$ and in MET by $10.9 \%$. Assuming normal distributions of REV values in each region, these reductions in the cancer nodules were highly statistically significant with the respective p-values: $3.9^{*} 10^{-17}$ (PTA), 5.5*10-4 (PTB) and $4.5^{*} 10^{-36}$ (MET). REV reduction indicates significant increase of the CS gene expression control in the cancer cells by the homeostatic mechanisms to confine the fluctuations of the expression levels within narrow intervals, as reported by us in other cancer studies $[38,52]$.

Interestingly, among the CS genes, PLCB1 (phospholipase C, beta 1) and RAC1 (ras-related C3 botulinum toxin substrate 1) were three times more variably expressed in PTA than in PTB. However 11 genes: CCR1/5/7 (chemokine (C-C motif) receptor 1/5/7), GRK6 (G protein-coupled receptor kinase 6), MAPK3 (mitogen-activated protein kinase 3) NCF1 (neutrophil cytosolic factor 1), PARD3 (par-3 family cell polarity regulator), PIK3CG (phosphatidylinositol-4,5-bisphosphate 3-kinase, catalytic subunit gamma), PRKCB (protein kinase C, beta), WAS (Wiskott-Aldrich syndrome), XCR1(chemokine (C motif) receptor 1 ) were three times more variably expressed in PTB than in PTA. The REV differences indicate again different strengths of the transcripts' abundance control in the two cancer nodules from the same kidney.

\subsection{Changes in gene networking}

We found that with respect to NOR, the number of significantly coordinately (synergistically + antagonistically) expressed chemokine signaling genes with VHL increased in PTA by $103.8 \%$, in PTB by $18.9 \%$ and in MET by $118,8 \%$. This result indicates not only that carcinogenesis reorganizes the gene networking, but that the network is different from one cancer nodule to another. Remodeling of the transcriptomic networks occurs in all genomic fabrics as well as in all genomic fabrics interplay. Figure 6 illustrates the changes in the expression coordination among the first 30 alphabetically ordered chemokine signaling genes.

For some pairs, the significant coordination was switched between synergistic and antagonistic as in the case of ADCY3-CCL5 (negative in NOR, positive in PTA), ADCY3-CCL28 (positive in PTA, negative in PTB), ADCY3-CCL4 (negative in NOR, positive in MET). These switches indicate significant remodeling of the genomic fabric of the functional pathway, with synergistically expressed partners (positive correlation) becoming antagonistically expressed (negative correlation) and vice-versa. For instance, owing to the positive correlation in PTA, the cancer-associated increase of the chemokine CCL28 abundance increases the abundance of the linked adenylate cyclase $A D C Y 3$ that regulates numerous pathways, including calcium signaling. By contrast, the negative correlation results in the chemokine increase reducing the abundance of the linked adenylate cyclase. Hence, correlation switches has important downstream consequences. 


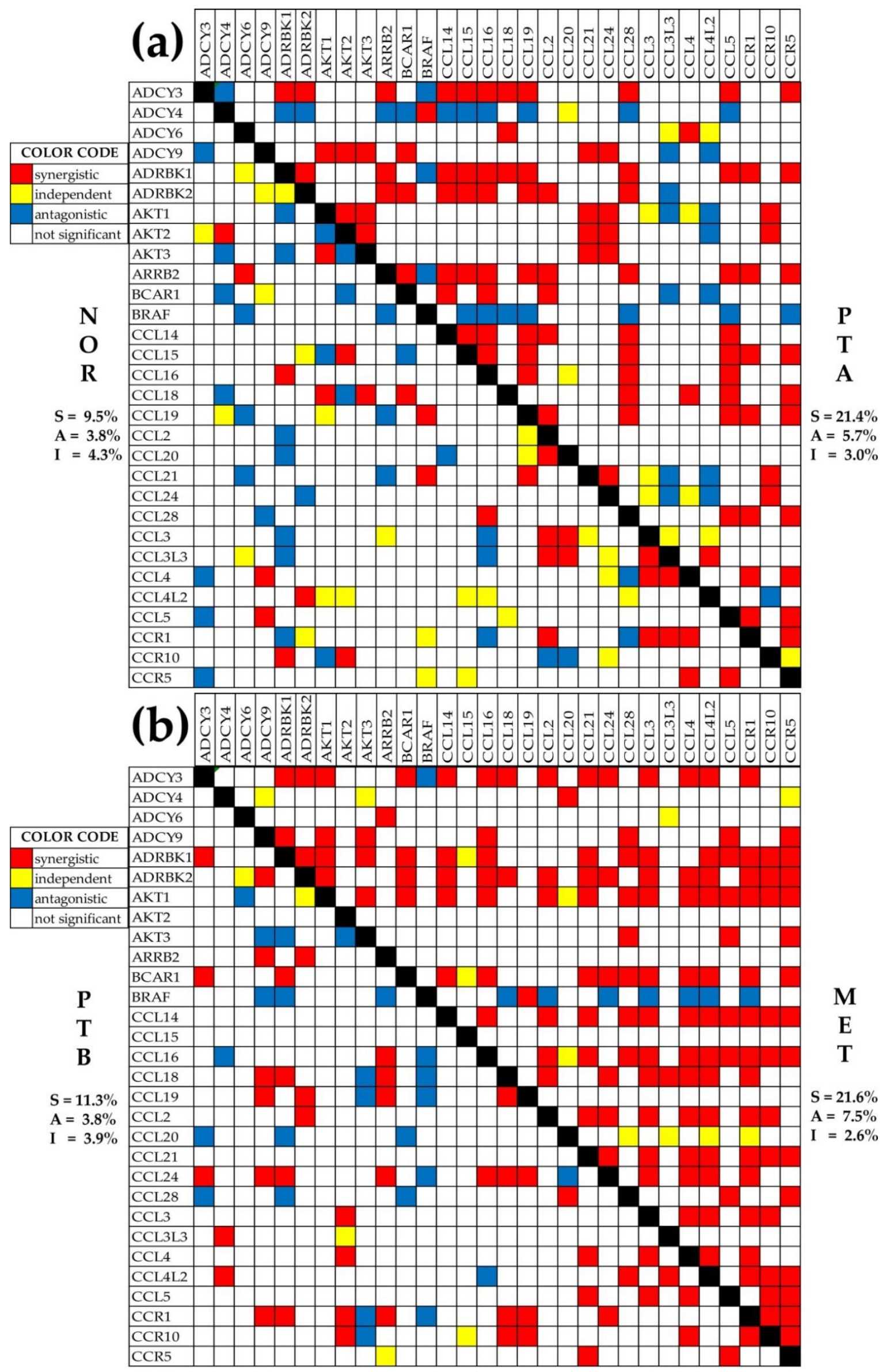


Figure 6 Metastatic ccRCC changes the expression coordination among the chemokine signaling genes. Red/blue/yellow color of a square indicates whether the genes labeling the intersecting row and column are significantly synergistically, antagonistically or independently expressed, while a blank square means that the correlation was not statistically significant. Numbers show the percentage of each type of coordination ( $\mathrm{S}$ = synergism, $\mathrm{A}=$ antagonism, $\mathrm{I}=$ independent) for the entire set of 130 chemokine signaling genes.

\subsection{Changes of the transcriptomic landscapes and genomic fabrics interplay}

In Figure 7 we used the Pair-Wise Relevance (PWR) analysis [53] to determine the transcriptomic landscapes of the genes responsible for the KEGG-determined oxidative phosphorylation pathway [54] in all four profiled regions. Note the effect of cancer in each region.

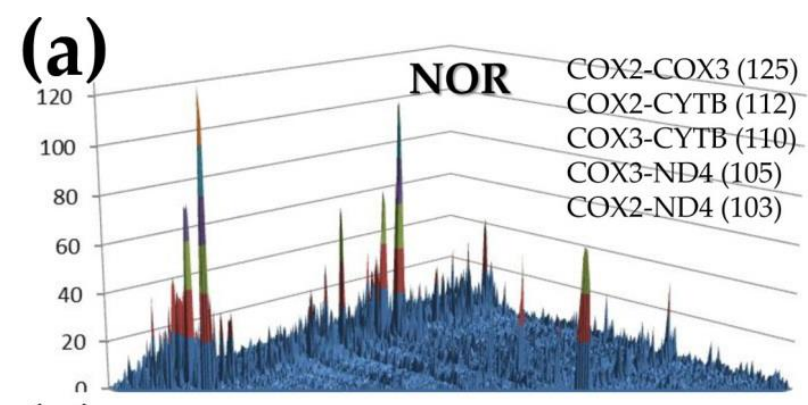

\section{(c)}
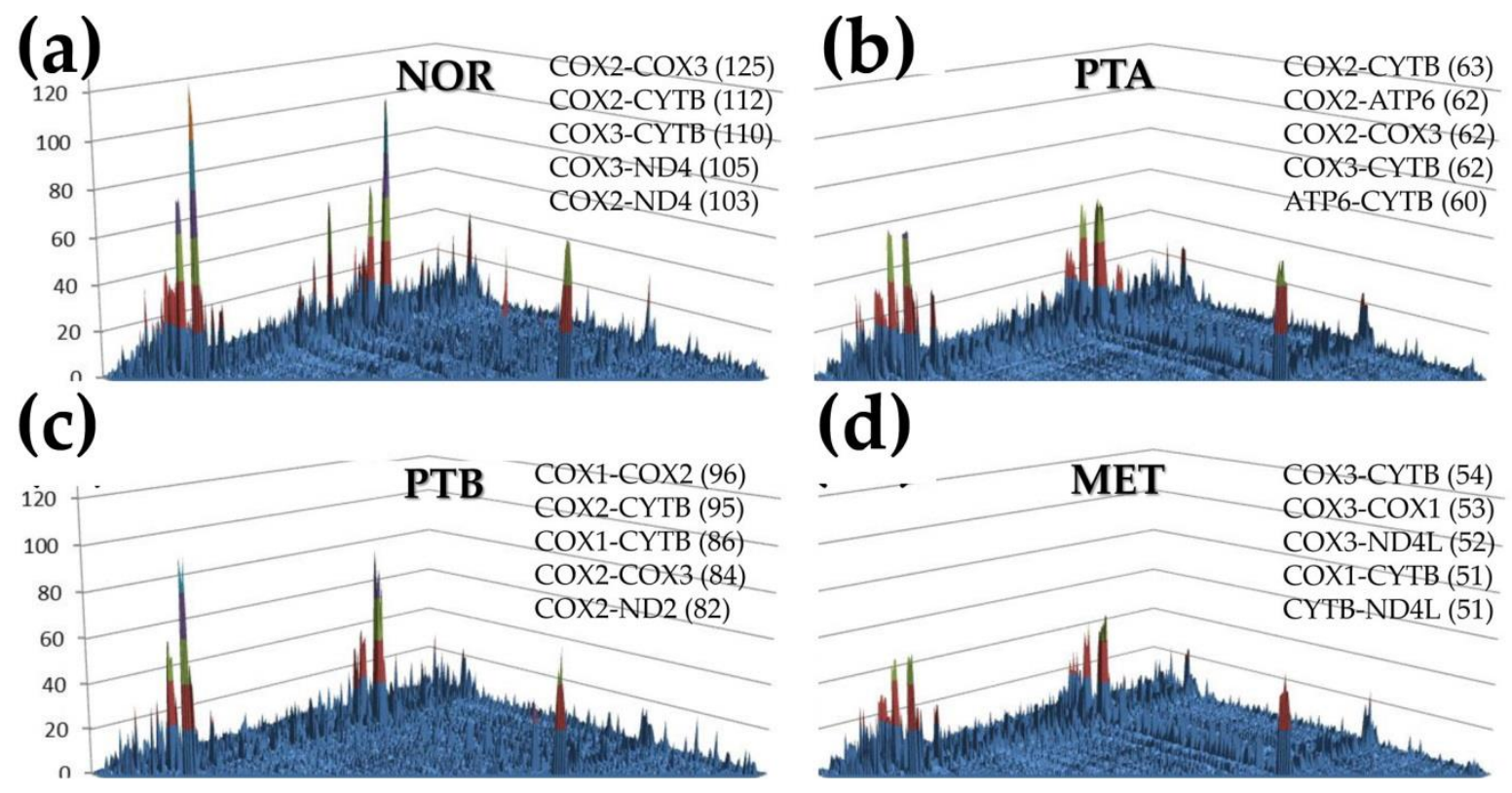

Figure 7 The PWR landscapes of mitochondrial genes in the four profiled regions. In each panel, the medallion lists the most relevant five pairs in that region. Genes of the most relevant pairs: ATP6 (mitochondrially encoded ATP synthase 6), COX1/2/3 (mitochondrially encoded cytochrome c oxidase I/2/3), CYTB (mitochondrially encoded cytochrome b), ND2/4/4L (mitochondrially encoded NADH dehydrogenase 2/4/4L).

PTA and MET have similar landscapes, while PTB is closer to NOR as resulted also from other above presented analyses and by comparing the percentages of the regulated genes in a previous study on the same dataset [32]. Interestingly, none of the genes from the listed most relevant 5 pairs in each region was regulated by cancer. We found that cancer not only remodels the transcriptomic landscapes of genomic fabrics (as illustrated in Fig. 7 for mitochondrial genes and in Ref [54] for oncogenes) but also strongly affects the interplay of the genomic fabrics of mitochondrial genes and oncogenes [55]. 


\subsection{Gene hierarchies and Gene Master Regulators}

We ranked the genes in each profiled region with respect to their Gene Commanding Height $(\mathrm{GCH})$ scores [56]. As illustrated in Figure 8, the gene hierarchy is strongly perturbed by the cancer. Note that each region has a different gene hierarchy (no overlap at least for the top 10 genes from Fig. 8a). For comparison, Fig. 8b presents the GCH scores of the 20 most frequently altered genes in KC. Remarkably, all of these biomarkers have poor scores in all regions profiled from this patient, far below the GMRs; the top ranked biomarker, MACF1 (microtubule-actin crosslinking factor 1), having $\mathrm{GCH}=11$ in PTA.

It is important to note that the GCH scores of cancer nodules GMRs (TASOR, FAM27C and $A L G 13)$ are very low in NOR, indicating that experimental manipulation of these GMRs may have disproportionally larger effects in the cancer regions than in NOR. Thus, targeting the GMR may offer a legitimate solution for the cancer gene therapy [38].

We found also that the GMRs of the four regions are located on various chromosomes (NOR-19, PTA-3, PTB-9, MET-X), and that they can transcribe in both coding (DAPK3, TASOR, ALG13) and non-coding (FAM27C) RNAs. Moreover, the GMRs of histopathologically distinct regions may be involved in different pathways. Interestingly, $D A P K 3$, the GMR of NOR, is part of the bladder cancer pathway [57]) and $A L G 13$, the GMR of MET, is active in the N-glycan biosynthesis [57]). For now, no functional pathway was assigned to TASOR and FAM27C, our study being the first to discuss their implication in the ccRCC. 
(a)

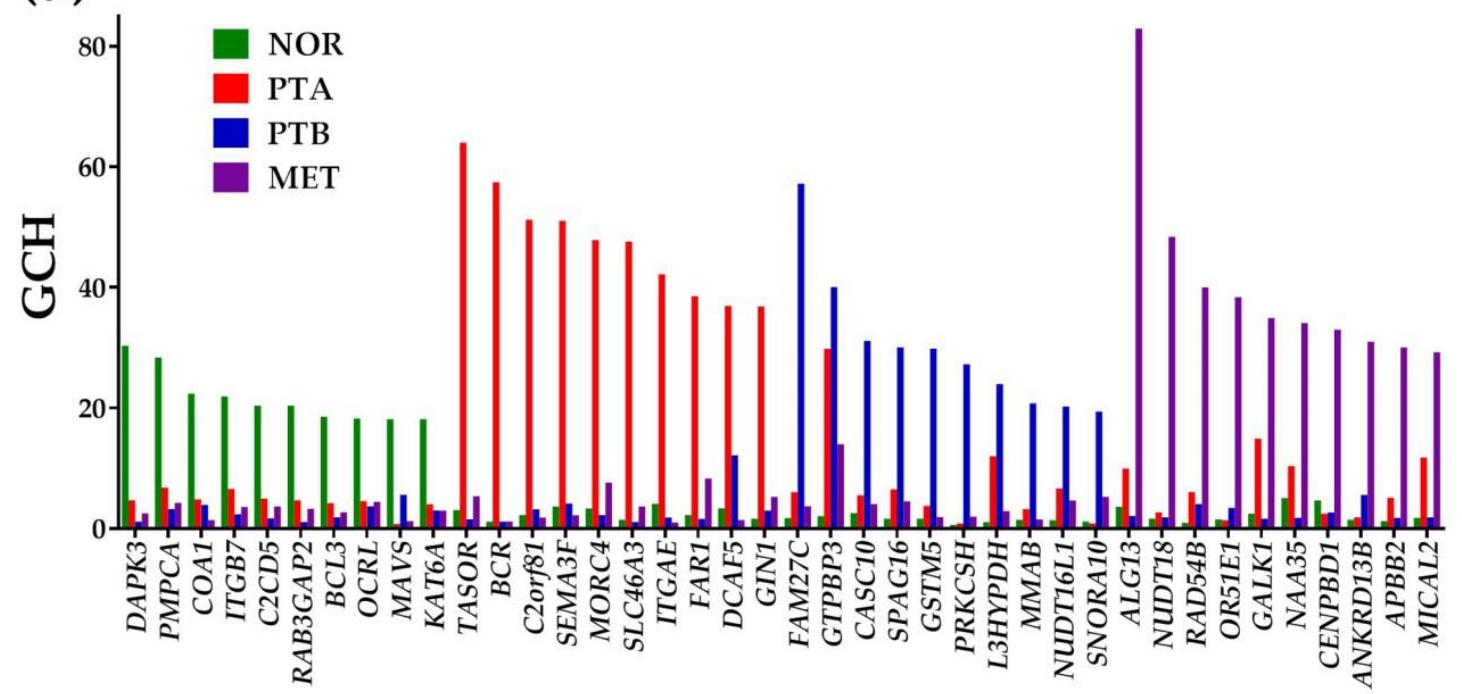

(b)

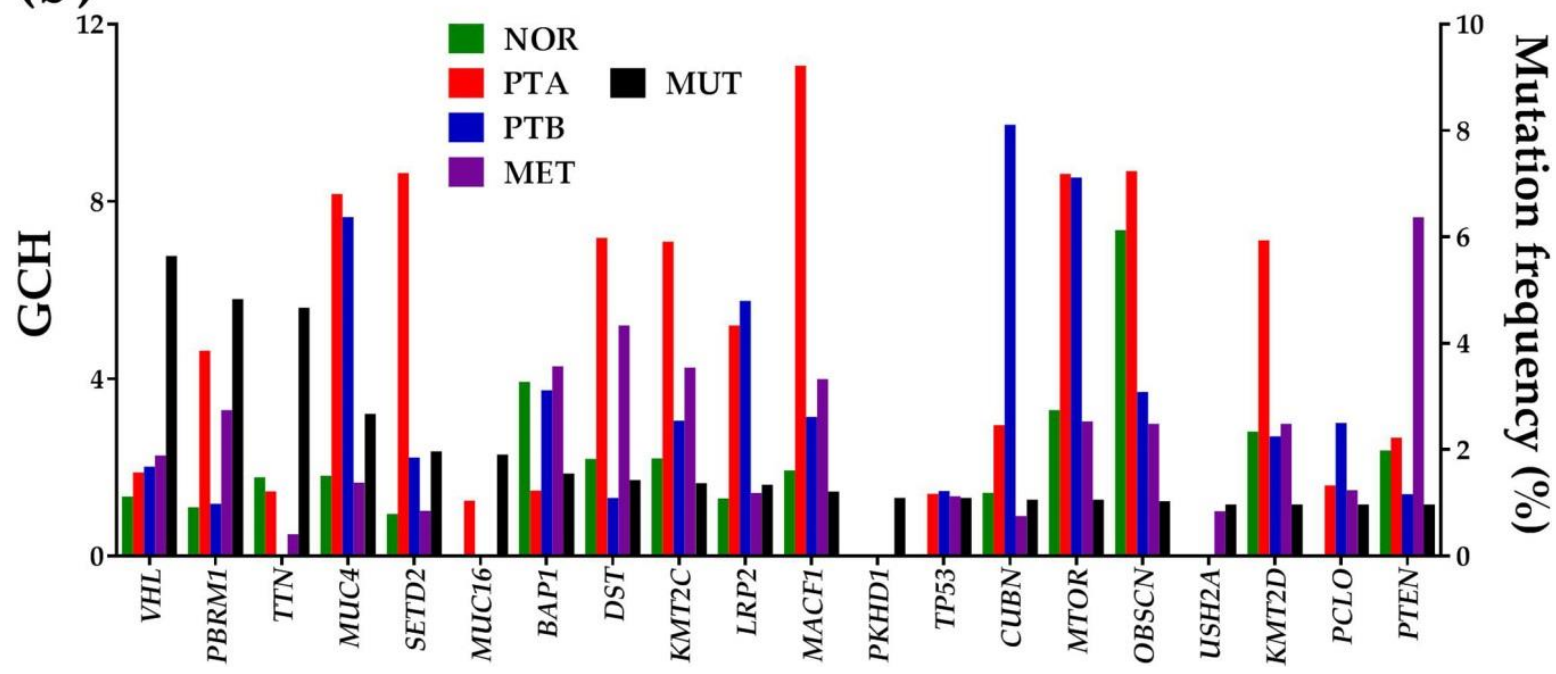

Figure 8 GCH scores of some remarkable genes in each profiled region. (a) Top 10 genes in each region and their GCH scores in the other regions. (b) GCH scores of the most frequently mutated genes in kidney cancer (mutation frequency on the right axis). Missing columns in (b) indicates that that gene was not adequately quantified in that region. GMRs: DAPK3 (death-associated protein kinase 3) from chromosome 19 in NOR, TASOR (transcription activation suppressor) from chromosome 3 in PTA, FAM27C (family with sequence similarity 27, member C, long non-coding RNA) from chromosome 9 in PTB and ALG13 (UDP-N-acetylglucosaminyltransferase subunit from chromosome $\mathrm{X}$ in MET.

\subsection{GMR at work}

Owing to the death of the patient who provided these samples soon after the surgery (and the lack of FDA approval for the GMR treatment) it was no way to validate the GMRs and determine their therapeutic values. However, we tried to delineate the molecular mechanisms by which the experimental manipulation of a cancer GMR selectively affects the cells it commands with minimal 
consequences for the normal cells. We used our previously reported finding that significant coordination of one gene with expression of other genes predicts with $>80 \%$ probability how the coordinately expressed genes will be regulated if the expression of the target gene is significantly altered [50]. This method was validated by stably transfecting two standard human thyroid cancer cell lines (BCPAP and 8505C) with four distinct genes (DDX19B, NEMP1, PANK2, UBALD1), and profiling their transcriptome before and after the transfection [37, 52].

Figure 9 presents possible action mechanisms for TASOR in PTA and ALG13 in MET, and the potential effects of the same GMRs in NOR. We did not yet figure a likely action mechanism for the non-coding FAM27C in PTB. Knowing that cancer cells have higher survival and growth rate than normal cells, we compared the expression levels and correlations with TASOR of the cellular transcriptional machinery in PTA and NOR. Because metastasis is characterized by uncontrollable cell division, we compared also the expression levels and with ALG13 of the cell-cycle (CC) pathway genes in MET and NOR.

We found that in PTA 15 out of 36 (42\%) quantified basal transcription factors [59] (BTF) and 14 out of $25(56 \%)$ genes from the RNA polymerase pathway [60] (POL) genes were ( $p>0.05)$ significantly antagonistically expressed with TASOR, the GMR of that region. No synergistic expression of TASOR with genes from these groups was identified (Fig. 9a). By contrast, in NOR there are $3 / 36(8 \%)$ BTF and 4/25 (16\%) POL genes synergistically expressed with TASOR but none antagonistically expressed. Therefore, we hypothesize that a significant overexpression of TASOR would down- regulate the antagonistically coordinated BTF and POL genes and up-regulate the synergistically expressed partners (Fig. 9b).

The treatment would correct the expression up-regulation of the BTF and POL genes in PTA, There are 12 up-regulated BTF genes in PTA: CDK9, ERCC3, GTF2F1, GTF2H1, GTF2H2C_2, GTF2H5, GTF2IRD1, TAF1, TAF10, TAF3, TAF6, TAF6L, and none down-regulated. In PTA there are also 8 upregulated POL genes: POLR1B, POLR1D, POLR2A, POLR2D, POLR2H, POLR2J, POLR2J2, POLR3GL but none down-regulated. These up-regulations explain the substantial higher growth rate in PTA with respect to NOR.

Interestingly, the statistically significant synergisms of TASOR with POLR1A and POLR2H in NOR are switched to significant antagonisms in PTA. Therefore, overexpression of TASOR is expected to have opposite consequences on these two genes in NOR (up-regulation) than in PTA (down-regulation). The up-regulation of 33\% of the quantified genes and the opposite correlations of TASOR with major transcription genes in PTA indicate that cancer profoundly affects the organization of the cellular transcription machinery.

In MET, the GMR ALG13 is synergistically expressed with $33(38 \%)$ and antagonistically expressed with 3 (3\%) of the 88 quantified KEGG-determined cell-cycle (CC) genes [61], while in NOR 3 (3\%) were synergistically and 4 (5\%) antagonistically expressed with ALG13 (Fig. 9c). Therefore, we hypothesize that knocking down $A L G 13$ will significantly slow-down the cell-cycle (hence cell proliferation) in MET by down-regulating the synergistically expressed CC genes. In NOR cells acceleration and deceleration caused by knocking down ALG13 will be practically balanced (Fig. 9d) owing to the 3:4 synergistic/antagonistic pairing with ALG13.

Again, genes such as: GADD45B (growth arrest and DNA-damage-inducible, beta), HDAC1 (histone deacetylase 1) and MCM3 (minichromosome maintenance complex component 3) were oppositely coordinated in the cancer nodule (MET) than in NOR. Therefore, it is expected that 
knocking down ALG13 will down-regulate these genes in MET while upregulating them in NOR. The knocking down of ALG13 would restore the normal CC pathway in MET affected by the upregulation of $25 \%$ of its genes ( $0 \%$ down-regulation). The upregulated $C C$ genes include the cyclins: CCNB1, CCNB1IP1, CCNC, CCND2, CCNY, CDK13, CDK2AP1, CDK4, CDK5, CDK5R1, CDK6, CDK9, CDKN2A, CDKN2D, CNNM3, GAK

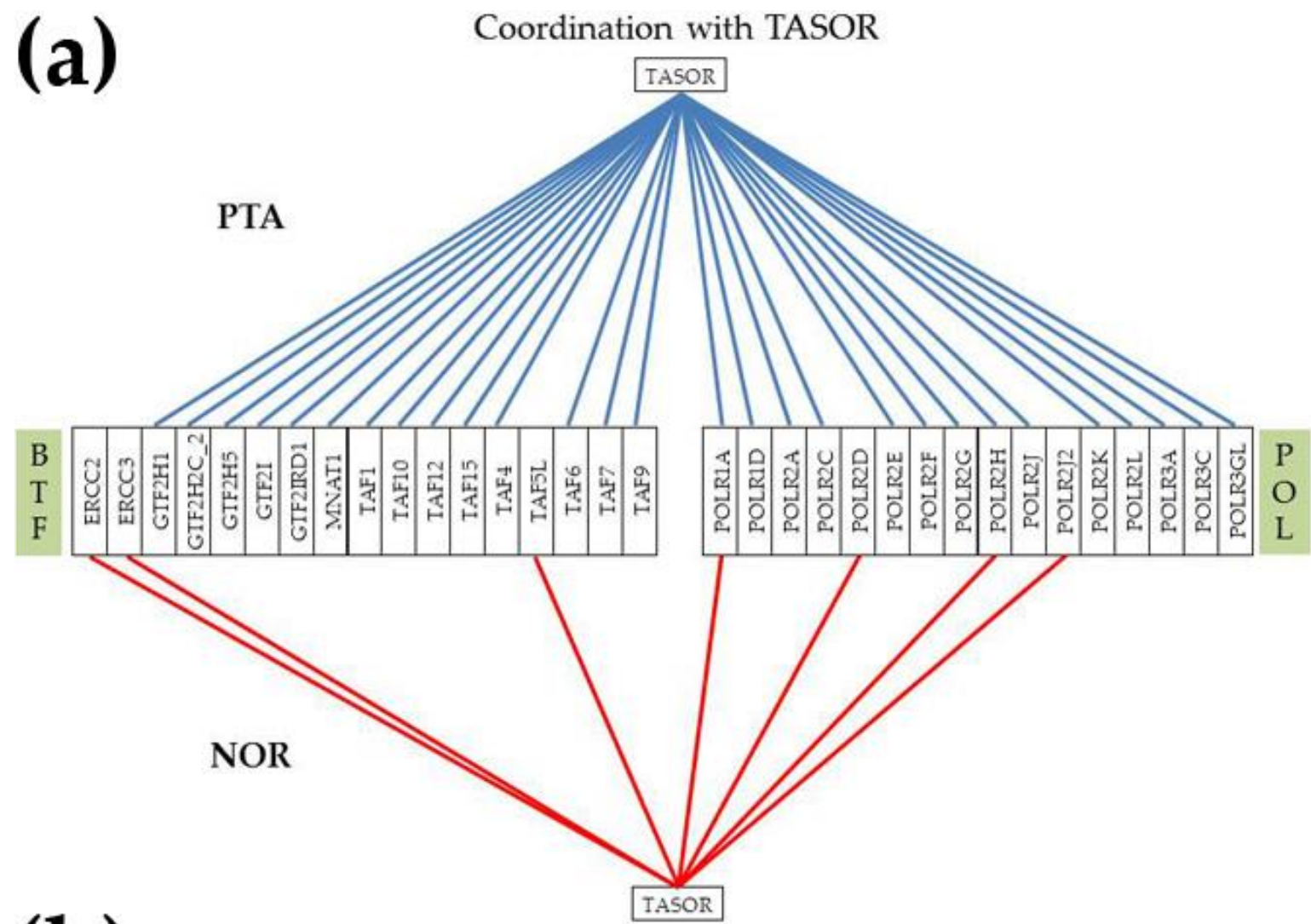

(b)

Regulation when TASOR is overexpressed

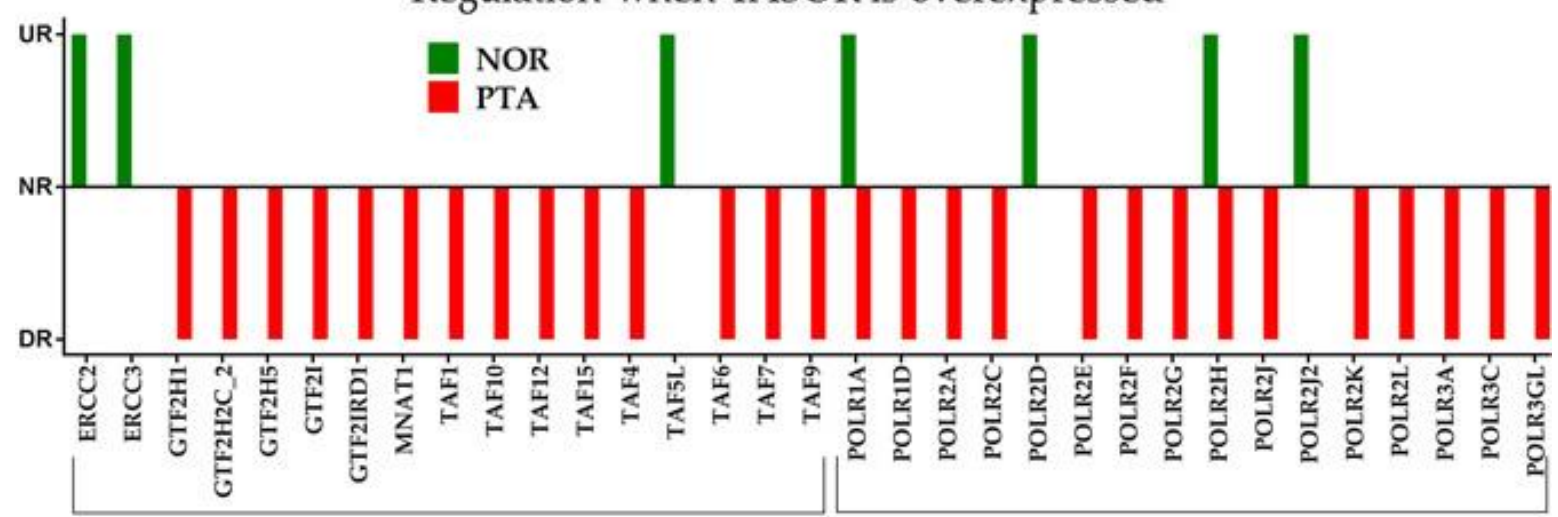

Basal transcription factors

RNA polymerase 


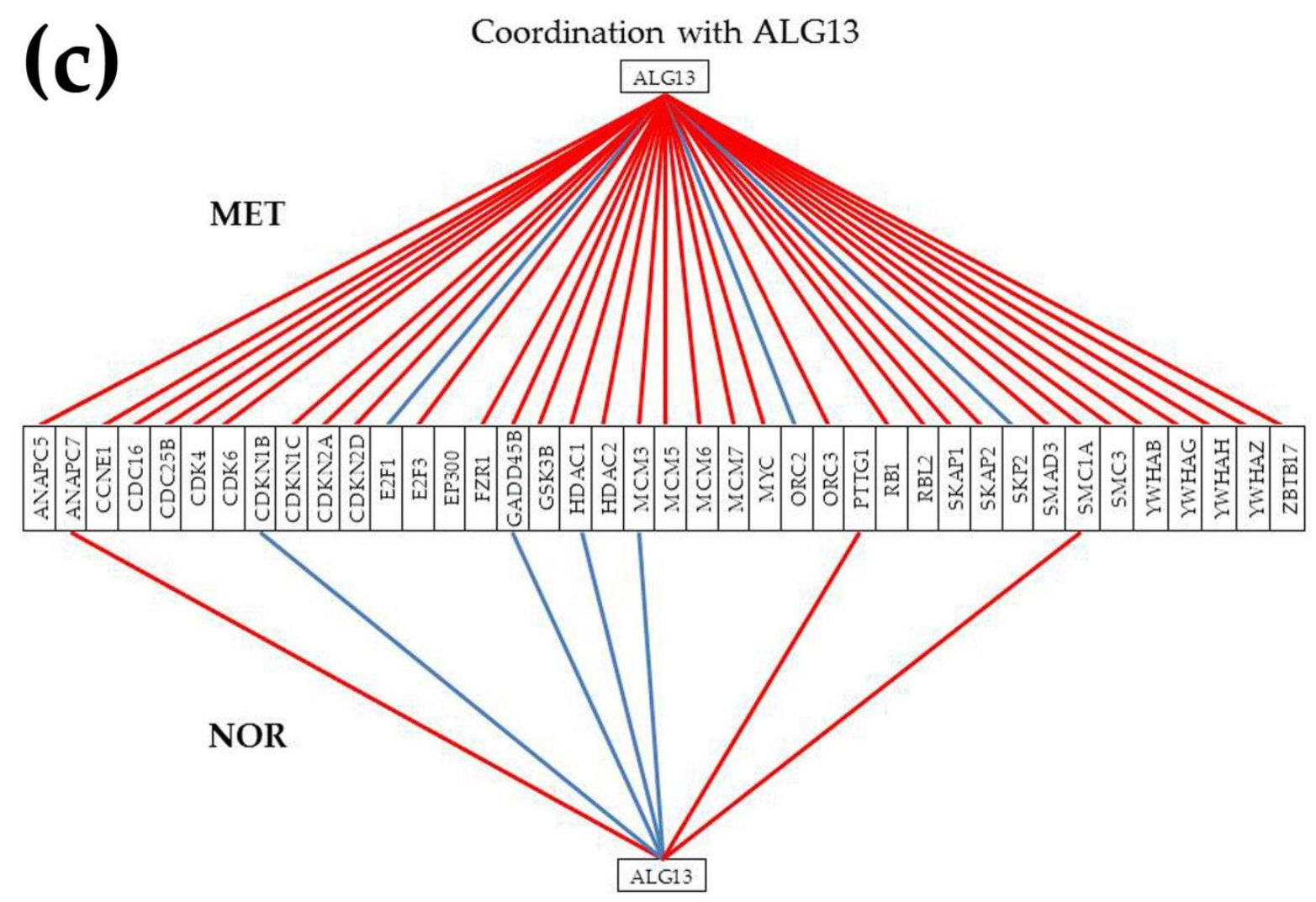

(d)

Regulation when ALG13 is knocked down

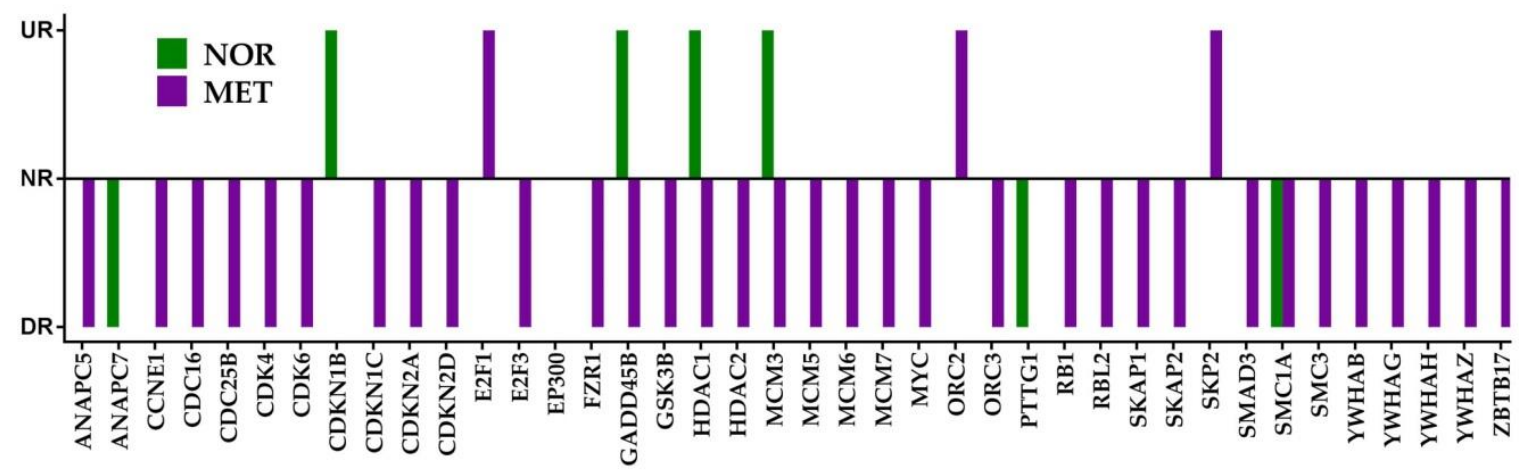

Figure 9 Expression correlation and predicted regulation of PTA, NOR and MET cells. (a-b) Effects of TASOR overexpression on the basal transcription factors (BFT) and polymerase pathway (POL) genes it correlates in PTA and NOR. (c-d) Effects of ALG13 knocking-down on the cell-cycle genes it correlates in MET and NOR.

\section{Discussion}

There are numerous (1,459 listed in PubMed [62]) studies using microarray data to identify altered gene expression profiles and biomarkers in ccRCC nodules with respect to normal counterparts (among the most recent [63-65]). One may ask: How are these findings a departure from other studies? The major difference is while other studies are unidimensional, relying only on the gene expression levels of the affected genes, ours is three-dimensional, increasing the available information by more than 4 orders of magnitude (number of distinct correlated partners of every 
gene). Moreover, gene expression profiles in cancer nodules were compared to their expressions in the surrounding normal tissue, the best reference to determine the cancer-related transcriptomic alterations.

In this report, we applied the Genomic Fabric Paradigm $[35,53,56]$ to the transcriptomic profiles of three cancer nodules and the surrounding healthy tissue surgically removed from a patient with metastatic kidney cancer. GFP relies on the three independent expression characteristics (average level, variability and correlation with expression of each other genes) that can be assigned to every quantified gene in each region. Previously, we have proven the independence of the three gene measures for the chemokine signaling genes in the normal kidney [55] and for the apoptosis genes in the papillary thyroid cancer nodule and surrounding normal tissue [52].

The most important and unique findings in our study are:

- In addition to regulating genes from several functional pathways (including apoptosis, VEGF and chemokine signaling), cancer also remodels the transcriptomic networks by which genes are influencing each-other expressions;

- Even histopathologically equally ranked cancer nodules in the same tumor have distinct transcriptomic organizations, alterations with respect to the normal surrounding tissue and gene hierarchies;

- Identification of TASOR, FAM27C and ALG13 as the GMRs of the three cancer nodules (PTA, PTB, MET) of the investigated person;

- GMRs of cancer nodules have low GCH scores in the normal tissue;

- Usual biomarkers have low GCH in both cancer and normal kidney tissue of this patient;

- Identification of molecular mechanisms by which overexpression of TASOR would substantially slow down proliferation of PTA cancer cells while stimulating regeneration of the normal ones;

- Identification of molecular mechanisms by which silencing of $A L G 3$ may block the cell cycle in MET without affecting it in NOR.

It is important to mention that, in each region, the median chemokine signaling gene had larger expression level than the median of all quantified genes in that region: with $7 \%$ in NOR, with $55 \%$ in PTA, with $34 \%$ in PTB and with $60 \%$ in MET. The higher expression indicates the importance of this pathway for the life of the renal cell. Moreover, as expected, compared to NOR, the median expression level of all 130 chemokine signaling genes was with $43 \%$ larger in PTA, with $13 \%$ larger in PTB and with 59\% larger in MET. The significant increase of the expression levels of chemokines in the cancer nodules is consistent with numerous reports about the chemokine role in cancer progression (e.g. [41, 42]). As mentioned in a previous study of the same dataset [32], the increase of the chemokine signaling is accompanied by increased cell proliferation through upregulation of cyclins and cyclin dependent kinases: 18 in PTA, 11 in PTB and 17 in MET. CCNB1IP1, CCNC, CCND2, $C D K 5 R 2, C D K 9$ and $G A K$ were up-regulated in all three regions. However, while no cyclin was downregulated in PTA and MET, five (CCNJL, CDK18, CDK19, CDK7, CCNM4) were down-regulated in PTB (6:5 up-/down-regulated).

The difference between the sets of the average expression levels in the two right kidney cancer nodules (PTA and PTB) was not statistically significant ( $\mathrm{p}$-val $=0.29$ ) owing to the large dispersion of the results. However, 25 (19\%) CS genes had significantly different expression levels between PTA and PTB. Moreover, the differences in REVs $(p-v a l=0.0003)$ and in correlation coefficients ( $p$-val = 0.0277) were statistically significant. The different REVs in Figure $1 \mathrm{~b}$ indicate different strengths of the cellular homeostatic control of transcript abundances among the profiled chemokines in each nodule and also differential control of the chemokine in distinct regions. Coordination analysis (Fig. 1c) revealed again differences among correlations of CS genes with VHL in the same nodule as well as differences between the two nodules for the same chemokine. The different expression correlations of $V H L$ in the two regions point to distinct gene networking [66], raising doubts about the universal structure (independent on race, age, sex, medical history etc.) of the KEGG-determined functional pathways. Altogether, the differences indicate distinct organizational principles [67] of the PTA and 
PTB transcriptomes. If this is the case for two regions with the same phenotype and isolated from the same kidney, how can one accept a meta-analysis comparing large populations of cancer and healthy patients?

A very interesting observation is the reduction of the expression variability in the cancer nodules with respect to the normal tissue. The statistical significance of the reduction increases considerably when larger numbers of genes are considered ( $p$-value $<10^{-100}$ for more than 2000 genes). Thus, when the analysis is applied to all 13,314 unredundantly quantified unigenes, the median REVs were: 41.51 (NOR), 31.45\% (PTA), 37.21\% (PTB) and 30.46\% (MET). These results confirmed our previous findings in numerous human and animal samples (cited in [56]), that a diseased tissue has a stricter control of the expression level (hence less variability). The robust result of increased expression control is presumably an evolutionary adaptation to limit the disease (here cancer)-related damages.

More than the differences between cancer and normal parts of the kidney, it is very important to point on the significant differences that we found between PTA and PTB, underlying the importance to adopt a therapeutic strategy that is tailored on each patient features [68]. In Fig. 2a we presented the separation of PTA and PTB with respect to two members of the adenylate cyclase family known to be associated with tumor progression and could serve as potential biomarkers for acute myeloid leukemia and other forms of cancer [69]. Since there are significant transcriptomic differences even between ccRCC nodules in the same kidney (illustrated in Fig. 2), the quest for transcriptomic signature of a particular form of cancer in all persons makes no sense. Another explanation for the transcriptomic differences among results obtained by various authors can be attributed to possible elevated RNA editing activity [70]. We believe that the RNA editing is sensitive to many local factors whose combination cannot be exactly repeated even in distinct histopathologicaly regions of the same tumor, much less in tumors of distinct patients that explains the tumor heterogeneity [71]. The differences between PTA and PTB were also evident in Figures 6a and $6 \mathrm{~b}$ showing the interconnection of chemokine signaling genes. We found also that the relevance of gene pairs are different in the two regions (Figures $7 \mathrm{~b}$ and 7c). Moreover, in Fig.8a we have shown that the two regions are governed by distinct of gene hierarchies, with no overlap among the top 10 genes.

Correlation analysis relies on the "Principle of Transcriptomic Stoichiometry" that requires genes whose encoded products are related in a functional pathway be coordinately expressed to optimize the pathway [36]. We used the coordination analysis to identify the molecular mechanisms by which manipulation of cancer GMRs may selectively affect the cancer cells. TASOR (a.k.a. FAM208A), the GMR of PTA, reported to be an important player for cell division [72], was synergistically correlated with POLR1A and POLR2H in NOR and antagonistically expressed with the same genes in PTA. Also, ALG13, an important modifier of renal filtration defects [73], was oppositely coordinated with GADD45B, HDAC1 and MCM3 in the cancer nodules with respect to NOR. These opposite coordinations indicate the strong dependence of the gene interactions on the cellular phenotype [56] and genetic background [21] that are disregarded by the functional pathways built by popular software (KEGG, Ingenuity, DAVID, GennMapp etc.).

Overexpression of TASOR is expected to have the following major effects in PTA:

- Down-regulation of three members of the general transcription factor $\mathrm{IIH}$, polypeptide (GTF2H1/2C_2/5); degradation of GTF2H1 (a.k.a. P62) was associated with enhanced apoptosis and autophagy [74].

- Down-regulation of the TATA-box binding protein associated factors: TAF1, TAF10, TAF12, TAF6, TAF7 and TAF9, all essential for the initiation of transcription by RNA polymerase II [75] and construct multi-protein complexes.

- Down-regulation of two components of RNA polymerase I complex (POLR1A, POLR1D) which transcribe DNA into ribosomal RNA (rRNA) precursors. The rRNA precursors are fundamental for ribosome biogenesis and protein synthesis and their inhibition limits cellular growth and proliferation [76]. 
- Down-regulation of nine components (POL2RA, POL2RC, POL2RE, POL2RF, POL2RG, POL2RH, POL2RJ, POL2RK, POL2RL) of the RNA polymerase II, responsible for synthesizing mRNA. It was recently reported that $P O L 2 R A$ silencing via siRNA is a treatment for triple negative breast cancer and induce a substantial reduction of tumor growth [77]. Our study shows that $P O L 2 R A$ could be also a target for ccRCC gene therapy.

- Down-regulation of three (POLR3A, POLR3C, POLR3GL) catalytic components of RNA polymerase III, which synthesize small RNAs and was suggested as a potential target for breast cancer [78].

By contrast, in NOR, overexpression of TASOR is expected to:

- Up-regulate $E R C C 2 / 3$ (excision repair cross-complementation group 2/3);

- Up-regulate TAF5L POLR1A, POLR2D, POLR2H, POLR2J

In summary, overexpression of TASOR would significantly reduce the transcription in cancer cells (hence their proliferation) while increasing the renewal of the normal cells.

Silencing ALG3 (via siRNA or CRISPR) would down-regulate numerous cell-cycle genes in MET, while keeping a relative balance in NOR. High expression of ALG13 was associated with poor overall survival in non-small-cell lung cancer [79]. Thus, silencing ALG13 would have both direct and indirect (mediated by CC genes) on the cancer cells. The subset of CC genes expected to be downregulated in MET includes:

- ANAPC5 (anaphase promoting complex subunit 5) that regulates cell cycle progression by ubiquitinating cell cycle proteins for proteolysis by the proteasome [80];

- One cyclin (CCNE1), two cell division cycles (CDC16, CDC25B), two cycle-dependent kinases (CDK4, CDK6), three cycle-dependent kinase inhibitors (CDKN1C, CDKN2A, CDKN2D);

- Four components of the minichromosome maintenance complex (MCM3, MCM5, MCM6, $M C M 7)$ and four tyrosine 3-monoxygenase/tryptophan 5-monoactivation proteins ( $Y W H A B$, YWHAG, YWHAH, YWHAZ).

All these genes are critical for the cell-cycle progression and their dysregulation may induce unbearable alterations of the cell transcriptome.

\section{Materials and Methods}

\subsection{Gene expression data}

In this study, we reanalyzed previously reported [32] gene expression data from a 74 years old male with metastatic ccRCC Fuhrman grade 3, who undergone total right kidney nephrectomy and resection of a chest wall mass. Publically available (own) raw and processed data were downloaded from [39]. The transcriptomic profiles were obtained by using Agilent $4 \times 44 \mathrm{~K}$ two-color microarray (platform GPL13497 [81]. Each sample collected from the two primary tumor nodules (PTA, PTB) and the surrounding normal tissue (NOR) in the right kidney, and the chest wall metastasis (MET) was split into four an each quarter profiled separately as a biological replicate (extensive protocol description in [32] and [39]. The results from NOR served as the reference for changes in the three cancer regions. Corrupted spots or with foreground fluorescence less than twice the background in at least one sample were discarded from analysis. Expression, variability and correlation of transcripts probed redundantly by several spots were averaged as described below (detailed presentation of the analyses can be found in [55])

\subsection{Average expression level (AVE)}

For each gene in each region we computed the average expression level of the four replicates considering the redundancy of the microarray spots probing the same gene, 
$\mu_{i}^{\text {(region })}=\frac{1}{R_{i}} \sum_{k=1}^{R_{i}} \mu_{i, k}^{\text {(region })}=\frac{1}{R_{i}} \sum_{k=1}^{R_{i}}\left(\frac{1}{4} \sum_{j=1}^{4} a_{i, k, j}^{\text {(region })}\right)$, where :

region $=$ NOR, $P T A, P T B, M E T$

$R_{i}=$ number of spots probing redundantly gene $i$

$a_{i, k, j}^{(\text {region })}=$ expression level of gene " $i$ " probed by spot " $k$ " on biological replica " $j$ " in "region"

\subsection{Relative Expression Variability}

Owing to the non-uniform redundancy of probing spots in the microarray, we replaced the coefficient of variation " $\mathrm{CV}$ " by the Bonferonni-like corrected mid-interval of the chi-square estimate of the pooled CV for all quantifiable transcripts of the same gene:

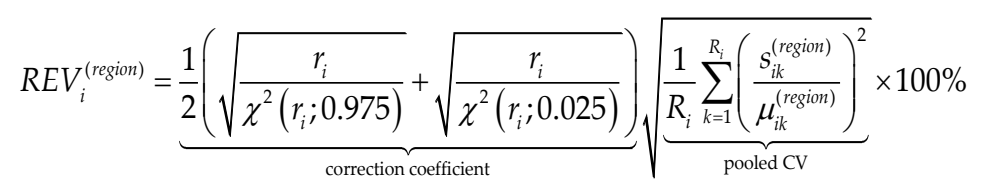

$\mu_{i k}=$ average expression level of gene $\mathrm{i}$ probed by spot $\mathrm{k}\left(=1, \ldots, R_{i}\right)$ in the 4 biological replicas

$s_{i k}=$ standard deviation of the expression level of gene i probed by spot $\mathrm{k}$

$r_{i}=4 R_{i}-1=$ number of degrees of freedom

$R_{i}=$ number of microarray spots probing redundantly gene $i$

\subsection{Expression coordination}

We computed the Pearson pair-wise correlation coefficient $\rho_{i j}$ between the expression levels of all pairs that could be formed with adequately quantified genes in each of the four regions.

\subsection{Expression regulation}

A gene was considered as significantly regulated in a cancer nodule (PTA, PTB, MET) with respect to NOR, or differentially expressed between the two primary tumors PTA and PTB if:

$\left|x_{i}^{(A \rightarrow B)}\right|>\operatorname{CUT}_{i}=1+\frac{1}{100} \sqrt{2\left(\left(R E V_{i}^{(A)}\right)^{2}+\left(R E V_{i}^{(B)}\right)^{2}\right)} \wedge p_{\text {val }}<0.05$

where:

$A=N O R, P T A, B=P T A, P T B, M E T$

$x_{i}^{(A \rightarrow B)} \equiv\left\{\begin{array}{ll}\frac{\mu_{i}^{(B)}}{\mu_{i}^{(A)}} \quad, \quad \text { if } \mu_{i}^{(B)}>\mu_{i}^{(A)} \\ -\frac{\mu_{i}^{(A)}}{\mu_{i}^{(B)}} \quad, \quad \text { if } \mu_{i}^{(B)}<\mu_{i}^{(A)}\end{array}, \quad \mu_{i}^{(A / B)}=\frac{1}{R_{i}} \sum_{k=1}^{R_{i}} \mu_{i k}^{(A / B)}\right.$

\subsection{Pair-Wise Relevance}

The pair-wise relevance (PWR) of genes $i$ and $j$ in the region $R=$ NOR, PTA, PTB, MET was computed as: 
$P W R_{i j}^{(R)}=\frac{\mu_{i}^{(R)} \mu_{j}^{(R)}}{\left(\overline{\mu^{(R)}}\right)^{2}} \times\left(\rho_{i j}^{(R)}\right)^{2} \times \frac{\left(\overline{R E V^{(R)}}\right)^{2}}{R E V_{i}^{(R)} R E V_{j}^{(R)}} \quad$, where:

$\overline{\mu^{(R)}}=\frac{1}{N} \sum_{k=1}^{N} \mu_{k}^{(R)} \quad, \quad N=$ number of unigenes,$\overline{R E V^{(R)}}=\frac{1}{N} \sum_{k=1}^{N} R E V_{k}^{(R)}$

$\rho_{i j}^{(R)}=$ Pearson correlation beween the expression levels of genes $i$ and $j$ in region $R$

4.7 Gene Commanding Height (GCH) and identification of Gene Master Regulator (GMR)

We have established the gene hierarchy in each region based on their GCH score:

$G C H_{i}^{(R)}=\underbrace{\frac{\langle R E V\rangle^{(R)}}{R E V_{i}^{(R)}}}_{\text {transcription control estimate }} \times \underbrace{\exp \left(\left.4 \overline{\left(\rho_{i j}^{(R)}\right)^{2}}\right|_{\forall j \neq i}\right)}_{\text {measure of expression coordination }}$, where :

\langle\rangle$=$ median, $\overline{()^{2}}=$ average of the square values

The GMR of region $R$ is the top gene (highest GCH) of that region.

\section{Conclusions}

The present study presents the principles of a new kind of systemic therapy of renal cancer metastasis [82] because by targeting the GMRs the cancer cells they command (and only them) would be attacked wherever they are in the body. Our personalized and time-sensitive approach that identifies the most suitable gene targets separately for each cancer nodule in every patient differs essentially from the popular quest for cancer biomarkers good for everybody (e.g. [83, 84]). This approach was possible because we went beyond the traditional analysis, limited to the average expression of individual genes only, by considering also their expression variability and expression correlation with each other gene.

The study has three major limitations:

i) We profiled heterogeneous tissues, composed of several cell types that diluted the transcriptomic alterations assigned to cancer cells. However, profiling separately each cell phenotype is not the solution. We proved by comparing the gene expression profiles of astrocytes and oligodendrocytes cultured alone and co-cultured in insert systems that the cell transcriptome is very sensitive to the cellular environment $[56,85]$. Thus, the transcriptome of the heterogeneous tissue is not the sum of the transcriptomes of the separately profiled individual cell types.

ii) Owing to the death of the patient and the lack of FDA approval, we had no possibility to test the procedure.

iii) Also, we had no possibility to perform additional molecular experiments for functional validation of the investigated pathways.

\section{References}

1. Key statistics about kidney cancer. Available on line: https://www.cancer.org/cancer/kidney-cancer.html. (accessed 8/2/2020)

2. McLaughlin, J.K.; Lipworth, L. Epidemiologic aspects of renal cell cancer. Semin Oncol. 2000; 27(2):115-23. PMID: 10768591.

3. TNM kidney cancer staging made simple. Available on line: https://kidneycancerinfo.weebly.com/stagingand-grading.html. (accessed 09/15/2020) 
4. Rioux-Leclercq, N. Le grade nucleaire de Fuhrman, facteur pronostique du cancer du rein depuis 25 ans [The Fuhrman grading system for kidney cancer prognosis]. Prog Urol. 2006; 16(4 Suppl FMC):5-8. French. PMID: 17183964.

5. Laskar, R.S.; Muller, D.C.; Li, P.; Machiela, M.J.; Ye, Y.; Gaborieau, V.; Foll, M.; Hofmann, J.N.; Colli, L.; Sampson, J.N. et al. Sex specific associations in genome wide association analysis of renal cell carcinoma. Eur J Hum Genet. 2019, 27(10):1589-1598. doi: 10.1038/s41431-019-0455-9. PMID: 31231134; PMCID: PMC6777615.

6. Pandey, J.; Syed, W. Cancer, Renal. In: StatPearls [Internet]. Treasure Island (FL, U.S.A.): StatPearls Publishing; 2020. PMID: 32644401. https://www.ncbi.nlm.nih.gov/books/NBK558975/ .

7. Purdue, M.P.; Song, L.; Scelo, G.; Houlston, R.S.; Wu, X.; Sakoda, L.C.; Thai, K.; Graff, R.E.; Rothman, N.; Brennan, P. et al. Pathway analysis of renal cell carcinoma genome-wide association studies identifies novel associations. Cancer Epidemiol Biomarkers Prev. 2020 30:cebp.0472.2020. doi: 10.1158/1055-9965.EPI-20-0472. PMID: 32732251.

8. Cui, H,; Xu, L.; Li, Z.; Hou, K.Z.; Che, X.F.; Liu, B.F.; Liu, Y.P.; Qu, X.J. Integrated bioinformatics analysis for the identification of potential key genes affecting the pathogenesis of clear cell renal cell carcinoma. Oncol Lett. 2020 20(2):1573-1584. doi: 10.3892/ol.2020.11703PMID: 32724399; PMCID: PMC7377202.

9. Tornberg, S.V.; Nisen, H.; Järvinen, P.; Järvinen, R.; Kilpeläinen, T.P.; Taari, K.; Stenman, U.H.; Visapää, H. Serum tumour associated trypsin inhibitor, as a biomarker for survival in renal cell carcinoma. Scand J Urol. 2020 4:1-7. doi: 10.1080/21681805.2020.1798501. PMID: 32748674.

10. Cancer Gov portal. Available on line: https://portal.gdc.cancer.gov (accessed 8/2/2020)

11. Ricketts, C.J.; De Cubas, A.A.; Fan, H.; Smith, C.C.; Lang, M.; Reznik, E.; Bowlby, R.; Gibb, E.A.; Akbani, R.; Cancer Genome Atlas Research Network et al. The Cancer Genome Atlas Comprehensive Molecular Characterization of Renal Cell Carcinoma. Cell Rep. 2018; 23(12):3698. doi: 10.1016/j.celrep.2018.06.032.

12. Linehan, W.M.; Schmidt, L.S.; Crooks, D.R.; Wei, D.; Srinivasan, R.; Lang, M.; Ricketts, C.J. The Metabolic Basis of Kidney Cancer. Cancer Discov. 2019; 9(8):1006-1021. doi: 10.1158/2159-8290.CD-18-1354. EPMID: 31088840 .

13. Linehan, W.M.; Walther, M.M.; Zbar, B. The genetic basis of cancer of the kidney. J Urol. 2003; 170(6 Pt 1):2163-72. doi: 10.1097/01.ju.0000096060.92397.ed. PMID: 14634372.

14. Mery, B.; Jones, S.; Vallard, A.; Rowinski, E.; Guillot, A.; Magné, N. Cancer du rein métastatique : recommandations et perspectives en 1re ligne. Bull Cancer. 2018; 105 Suppl 3:S235-S241. French. doi: 10.1016/S0007-4551(18)30378-3. PMID: 30595152.

15. Spirina, L.V.; Yurmazov, Z.A.; Gorbunov, A.K.; Usynin, E.A.; Lushnikova, N.A.; Kovaleva, I.V. Molecular Protein and Expression Profile in the Primary Tumors of Clear Cell Renal Carcinoma and Metastases. Cells. 2020; 9(7):E1680. doi: 10.3390/cells9071680. PMID: 32668608.

16. Hu C, Li L, Ding P, Li L, Ge X, Zheng L, Wang X, Wang J, Zhang W, Wang N. et al. Complement Inhibitor CRIg/FH Ameliorates Renal Ischemia Reperfusion Injury via Activation of PI3K/AKT Signaling. J Immunol. 2018 Dec 15;201(12):3717-3730. doi: 10.4049/jimmunol.1800987. Epub 2018 Nov 14. PMID: 30429287; PMCID: PMC6287101.

17. Al Kafri, N.; Hafizi, S. Tumour-Secreted Protein S (ProS1) Activates a Tyro3-Erk Signalling Axis and Protects Cancer Cells from Apoptosis. Cancers (Basel). 2019; 11(12):1843. doi: 10.3390/cancers11121843. PMID: 31766614; PMCID: PMC6966665.

18. Gasinska, A.; Jaszczynski, J.; Adamczyk, A.; Janecka-Widła, A.; Wilk, W.; Cichocka, A.; Stelmach, A. Biomarkers of epithelial-mesenchymal transition in localized, surgically treated clear-cell renal cell carcinoma. Folia Histochem Cytobiol. 2018;56(4):195-206. doi: 10.5603/FHC.a2018.0023. Epub 2018 Dec 20. PMID: 30569446.

19. Alcaraz, E.; Vilardell, J.; Borgo, C.; Sarró, E.; Plana, M.; Marin, O.; Pinna, L.A.; Bayascas, .J.; Meseguer, A.; Salvi, M. et al. Effects of CK2 $\beta$ subunit down-regulation on Akt signalling in HK-2 renal cells. PLoS One. 2020; 15(1):e0227340. doi: 10.1371/journal.pone.0227340. PMID: 31910234; PMCID: PMC6946142.

20. Park, J.Y.; Lin, P.Y.; Weiss, R.H. Targeting the PI3K-Akt pathway in kidney cancer. Expert Rev Anticancer Ther. 2007; 7(6):863-70. doi: 10.1586/14737140.7.6.863. PMID: 17555396.

21. Iacobas, S.; Iacobas, D.A.; Spray, D.C.; Scemes, E. The connexin 43 transcriptome during brain development: importance of genetic background. Brain Research 2012, 1487: 131-139. doi: 10.1016/j.brainres.2012.05.062. PMCID:PMC3501561. 
22. Thomas, N.M.; Jasmin, J.F.; Lisanti, M.P.; Iacobas, D.A. Sex Differences in Expression and subcellular Localization of Heart Rhythm Determinant Proteins. Biochem Biophys Res Commun 2011, 406(1):117-22. doi: 10.1016/j.bbrc.2011.02.006. PMID:21296051.

23. Iacobas, D.A.; Fan, C.; Iacobas, S.; Spray, D.C.; Haddad, G.G. Transcriptomic changes in developing kidney exposed to chronic hypoxia. Biochem Biophys Res Comm 2006, 349(1), 329-338. DOI:10.1016/j.bbrc.2006.08.056. PMID:16934745.

24. Desruisseaux, M.; Iacobas, D.A.; Iacobas, S.; Mukherjee, S.; Weiss, L.M.; Tanowitz, H.B.; Spray, D.C. Alterations in the Brain Transcriptome in Plasmodium Berghei ANKA Infected. J Neuroparasitology 2010, 1: 74-81. PMCID: PMC3587055.

25. Iacobas, D.A.; Iacobas, S.; Nebieridze, N.; Velisek, L.; Veliskova, J. Estrogen protects neurotransmission transcriptome during status epilepticus, Front Neurosci 2018, 12:332. DOI: 18.3389/fnins.2018.00332.

26. Fan, C.; Iacobas, D.A.; Zhou, D.; Chen, Q.; Gavrialov, O.; Haddad, G.G. Gene expression and phenotypic characterization of mouse heart after chronic constant and intermittent hypoxia. Physiol Genomics 2005, 22: 292-307. DOI: 10.1152/physiolgenomics.00217.2004. PMCID:PMC2856928.

27. Thi. M.M.; Iacobas, D.A.; Iacobas, S.; Spray, D.C. Fluid shear stress regulates vascular endothelial growth factor gene in osteoblasts. Ann N Y Acad Sci 2007, 1117: 73-81. DOI: 10.1196/annals.1402.020. PMID:17646268.

28. Kobets, T.; Iatropoulos, M.J.; Duan, J.D,; Brunnemann, K.D.; Iacobas, D.A.; Iacobas, S.; Vock, E.; Deschl, U.; Williams, G.M. Effects of Nitrosamines on the Expression of Genes Involved in Xenobiotic Metabolism in the Chicken Egg Alternative Genotoxicity Model. Toxicol Sci 2018, 166(1), 82-96, doi: 10.1093/toxsci/kfy197. PMID: 30102407.

29. Iacobas, D.A.; Chachua, T.; Iacobas, S.; Benson, M.J.; Borges, K.; Veliskova, J.; Velisek, L. ACTH and PMX53 recover the normal synaptic transcriptome in a rat model of infantile spasms. Sci Rep 2018. 8:5722, PMCID: PMC5893534. DOI:10.1038/s41598-018-24013-x.

30. Lee, P.R.; Cohen, J.E.; Iacobas, D.A.; Iacobas, S.; Fields, R.D. Gene networks activated by pattern-specific generation of action potentials in dorsal root ganglia neurons. Sci Rep 2017, 7:43765, doi:10.1038/srep43765.

31. Dwivedi, D.K.; Xi, Y.; Kapur, P.; Madhuranthakam, A.J.; Lewis, M.A.; Udayakumar, D.; Rasmussen, R.; Yuan, Q.; Bagrodia, A.; Margulis, V. et al. Magnetic Resonance Imaging Radiomics Analyses for Prediction of High-Grade Histology and Necrosis in Clear Cell Renal Cell Carcinoma: Preliminary Experience. Clin Genitourin Cancer 2020, S1558-7673(20)30119-1. doi: 10.1016/j.clgc.2020.05.011. PMID: 32669212.

32. Iacobas, D.A.; Iacobas, S. Towards a Personalized Cancer Gene Therapy: A Case of Clear Cell Renal Cell Carcinoma. Cancer \& Oncol Res 2017, 5(3): 45-52. DOI:10.13189/cor.2017.050301.

33. Noon, A. P.; Vlatković, N.; Polański, R.; Maguire, M.; Shawki, H.; Parsons, K.; Boyd, M. T. p53 and MDM2 in renal cell carcinoma: biomarkers for disease progression and future therapeutic targets?. Cancer 2010, 116(4), 780-790. https://doi.org/10.1002/cncr.24841.

34. Harlander, S.; Schönenberger, D.; Toussaint, N. C.; Prummer, M.; Catalano, A.; Brandt, L.; Moch, H.; Wild, P. J.; Frew, I. J. Combined mutation in Vhl, Trp53 and Rb1 causes clear cell renal cell carcinoma in mice. Nature medicine 2017, 23(7), 869-877. https://doi.org/10.1038/nm.4343.

35. Iacobas, D.A. The Genomic Fabric Perspective on the Transcriptome between Universal Quantifiers and Personalized Genomic Medicine. Biological Theory 2016, 11(3): 123-137. DOI 10.1007/s13752-016-0245-3.

36. Iacobas, D.A.; Iacobas, S.; Lee, P.R.; Cohen, J.E.; Fields, R.D. Coordinated Activity of Transcriptional Networks Responding to the Pattern of Action Potential Firing in Neurons. Genes (Basel) 2019, 10(10), 754. PMID: 31561430, Doi: 10.3390/genes10100754.

37. Iacobas, D.A.; Tuli, N.; Iacobas, S.; Rasamny, J.K.; Moscatello, A.; Geliebter, J.; Tiwari, R.M. Gene master regulators of papillary and anaplastic thyroid cancer phenotypes. Oncotarget 2018, 9(2), 2410-2424. doi: 10.18632/oncotarget.23417. PMCID: PMC5788649.

38. Iacobas, S.; Ede, N.; Iacobas, D.A. The Gene Master Regulators (GMR) Approach Provides Legitimate Targets for Personalized, Time-Sensitive Cancer Gene Therapy. Genes (Basel) 2019, 10(8), 560. doi:10.3390/genes10080560.

39. Remodeling of major genomic fabrics and their interplay in metastatic clear cell renal cell carcinoma. Available online: https://www.ncbi.nlm.nih.gov/geo/query/acc.cgi?acc=GSE72304 (accessed 06/02/2020).

40. Chemokine signaling pathway. Available online: https://www.kegg.jp/keggbin/show_pathway?hsa04062map hsa04062 (accessed 07/03/2020). 
41. Hussain, M.; Adah, D.; Tariq, M.; Lu, Y.; Zhang, J.; Liu, J. CXCL13/CXCR5 signaling axis in cancer. Life Sci. 2019; 227:175-186. doi: 10.1016/j.lfs.2019.04.053. Epub 2019 Apr 23. PMID: 31026453.

42. Kadomoto, S.; Izumi, K.; Mizokami, A. The CCL20-CCR6 Axis in Cancer Progression. Int J Mol Sci. 2020; 21(15):5186. doi: 10.3390/ijms21155186. PMID: 32707869; PMCID: PMC7432448.

43. Hsieh, J.J.; Cheng, E.H. Exploiting the circuit breaker cancer evolution model in human clear cell renal cell carcinoma. Cell Stress 2020, 4(8):191-198. doi: 10.15698/cst2020.08.227. PMID: 32743344; PMCID: PMC7380452.

44. Renal Cell Carcinoma pathway. Available online: https://www.kegg.jp/keggbin/show pathway?hsa05211. (accessed 07/03/2020).

45. Prickett, D.; Watson, M. Use of GenMAPP and MAPPFinder to analyse pathways involved in chickens infected with the protozoan parasite Eimeria. BMC Proc 20093 Suppl 4: S7. PMCID: PMC2712750 DOI: 10.1186/1753-6561-3-S4-S7

46. Apoptosis pathway. Available on line: https://www.kegg.jp/kegg-bin/show pathway?hsa04210 (accessed $\underline{06 / 03 / 2020)}$.

47. VEGF signaling pathway. Available online:_https://www.kegg.jp/kegg-bin/show_pathway?hsa04370. (accessed 07/03/2020).

48. Rini, B.I.; Battle, D.; Figlin, R.A.; George, D.J.; Hammers, H.; Hutson, T.; Jonasch, E.; Joseph, R.W.; McDermott, D.F.; Motzer, R.J. et al. The society for immunotherapy of cancer consensus statement on immunotherapy for the treatment of advanced renal cell carcinoma (RCC). J Immunother Cancer. 2019; 7(1):354. doi: 10.1186/s40425-019-0813-8. PMID: 31856918; PMCID: PMC6924043.

49. Melincovici, C.S.; Boşca, A.B.; Şuşman, S.; Mărginean, M.; Mihu, C.; Istrate, M.; Moldovan, I.M.; Roman, A.L.; Mihu, C.M. Vascular endothelial growth factor (VEGF) - key factor in normal and pathological angiogenesis. Rom J Morphol Embryol. 2018; 59(2):455-467. PMID: 30173249.

50. Yarla, N.S.; Bishayee, A.; Vadlakonda, L.; Chintala, R.; Duddukuri, G.R.; Reddanna, P.; Dowluru, K.S. Phospholipase A2 Isoforms as Novel Targets for Prevention and Treatment of Inflammatory and Oncologic Diseases. Curr Drug Targets. 2016; 17(16):1940-1962. doi: 10.2174/1389450116666150727122501. PMID: 26212262.

51. Li, S.; Jiang, M.; Wang, L.; Yu, S. Combined chemotherapy with cyclooxygenase-2 (COX-2) inhibitors in treating human cancers: Recent advancement. Biomed Pharmacother. 202012; 129:110389. doi: 10.1016/j.biopha.2020.110389. PMID: 32540642.

52. Iacobas, D.A. Biomarkers, master regulators and genomic fabric remodeling in a case of papillary thyroid carcinoma. Genes (Basel) 2020, 11(9), 1030. https://doi.org/10.3390/genes11091030

53. Iacobas, S.; Thomas, N.M.; Iacobas, D.A. Plasticity of the myelination genomic fabric. Mol Genet Genomics 2012 287:237-246. https://doi.org/10.1007/s00438-012-0673-0. PMID: 22246408.

54. Oxidative phosphorylation pathway. Available on line: https://www.kegg.jp/keggbin/show_pathway?hsa00190 (accessed 08/20/2020).

55. Iacobas, D.A. Powerful quantifiers for cancer transcriptomics. World J Clin Oncol 2020; 11(9): 679-704, https://www.wjgnet.com/2218-4333/full/v11/i9/679.htm.

56. Iacobas, D.A.; Iacobas, S.; Stout, R.; Spray, D.C. Cellular environment remodels the genomic fabrics of functional pathways in astrocytes. Genes (Basel) 2020 11(5), 520; PMID: 32392822, doi: 10.3390/genes11050520.

57. Bladder cancer pathway. Available online: https://www.kegg.jp/kegg-bin/show_pathway?hsa05219+1613 (accessed 09/17/2020).

58. N-glycan biosynthesis. Available online: https://www.kegg.jp/kegg-bin/show_pathway?hsa00510+79868 (accessed 09/17/2020)

59. Basal transcription factors. Available online: https://www.kegg.jp/kegg-bin/show_pathway?hsa03022 (accessed 09/20/2020)

60. RNA polymerase pathway. Available on line: https://www.kegg.jp/kegg-bin/show_pathway?hsa03020 (accessed 09/20/2020).

61. Cell cycle pathway. Available on line: https://www.kegg.jp/kegg-bin/show_pathway?hsa04110 (accessed 09/20/2020).

62. NCBI PubMed microarray studies on renal cancers. Available on line: https://pubmed.ncbi.nlm.nih.gov/?term=renal+cancer\%2C+microarray\&sort=date (accessed 09/26/2020). 
63. Cui, Y.; Miao, C.; Hou, C.; Wang, Z.; Liu B. Apolipoprotein C1 (APOC1): A Novel Diagnostic and Prognostic Biomarker for Clear Cell Renal Cell Carcinoma. Front Oncol. 2020 10:1436. doi: 10.3389/fonc.2020.01436. PMID: 32974161; PMCID: PMC7468425.

64. Ma, C.G.; Xu, W.H.; Xu, Y.; Wang, J.; Liu, W.R.;, Cao, D.L.; Wang, H.K.; Shi, G.H.; Zhu. Y.P.; Qu, Y.Y.; et al. Identification and validation of novel metastasis-related signatures of clear cell renal cell carcinoma using gene expression databases. Am J Transl Res. 2020 12(8):4108-4126. PMID: 32913492; PMCID: PMC7476160.

65. Allen, A.; Gau, D.; Francoeur, P.; Sturm, J.; Wang, Y.; Martin, R.; Maranchie, J.; Duensing, A.; Kaczorowski, A.; Duensing, S.; et al. Actin-binding protein Profilin1 promotes aggressiveness of clear cell renal cell carcinoma cells. J Biol Chem. 2020 jbc.RA120.013963. doi: 10.1074/jbc.RA120.013963. PMID: 32883810.

66. Iacobas, D.A.; Iacobas, S.; Werner, P.; Scemes, E.; Spray, D.C. Alteration of transcriptomic networks in adoptive-transfer experimental autoimmune encephalomyelitis. Front Integr Neurosci 2007, 1:10. doi:10.3389/neuro.07/010.2007. PMCID: PMC2526015.

67. Spray, D.C.; Iacobas, D.A. Organizational principles of the connexin-related brain transcriptome. J Membr Biol. 2007 218(1-3):39-47. DOI:10.1007/s00232-007-9049-5. PMID:17657523.

68. di Martino, S.; De Luca, G.; Grassi, L.; Federici, G.; Alfonsi, R.; Signore, M.; Addario, A.; De Salvo, L.; Francescangeli, F.; Sanchez, M. et al. Renal cancer: new models and approach for personalizing therapy. J Exp Clin Cancer Res. 2018 37(1):217. doi: 10.1186/s13046-018-0874-4.

69. Chen, S.L.; Hu, F.; Wang, D.W.; Qin, Z.Y.; Liang, Y/; Dai, Y.J. Prognosis and regulation of an adenylyl cyclase network in acute myeloid leukemia. Aging (Albany NY). 2020; 12(12):11864-11877. doi: 10.18632/aging.103357. PMID: 32568101; PMCID: PMC7343484.

70. Barth, D.A.; Slaby, O.; Klec, C.; Juracek, J.; Drula, R.; Calin, G.A.; Pichler, M. Current Concepts of NonCoding RNAs in the Pathogenesis of Non-Clear Cell Renal Cell Carcinoma. Cancers (Basel). 2019; 11(10):1580. doi: 10.3390/cancers11101580. PMID: 31627266; PMCID: PMC6826455.

71. Paz-Yaacov, N.; Bazak, L.; Buchumenski, I.; Porath, H.T.; Danan-Gotthold, M.; Knisbacher, B.A.; Eisenberg, E.; Levanon, E.Y. Elevated RNA Editing Activity Is a Major Contributor to Transcriptomic Diversity in Tumors. Cell Rep. 2015; 13(2):267-76. doi: 10.1016/j.celrep.2015.08.080. Epub 2015 Oct 1. PMID: 26440895.

72. Gresakova, V.; Novosadova, V.; Prochazkova, M.; Bhargava, S.; Jenickova, I.; Prochazka, J.; Sedlacek, R. Fam208a orchestrates interaction protein network essential for early embryonic development and cell division. Exp Cell Res. 2019; 382(1):111437. doi: 10.1016/j.yexcr.2019.05.018. PMID: 31112734.

73. Esposito, T.; De Stefano, G.; Reccia, M.G.; Di Lorenzo, I.; Napolitano, F.; Scalabrì, F.; Lombardi, A.; Saleem, M.A.; Griffiths, L.R.; Gianfrancesco, F. Dysregulation of the Expression of Asparagine-Linked Glycosylation 13 Short Isoform 2 Affects Nephrin Function by Altering Its N-Linked Glycosylation. Nephron. 2017; 136(2):143-150. doi: 10.1159/000455129. PMID: 28178702.

74. Ji, J.; Zhou, X.; Xu, P.; Li, Y.; Shi, H.; Chen, D.; Li, R.; Shi, H. Deficiency of apoptosis-stimulating protein two of p53 ameliorates acute kidney injury induced by ischemia reperfusion in mice through upregulation of autophagy. J Cell Mol Med. 2019; 23(4):2457-2467. doi: 10.1111/jcmm.14094. PMID: 30675758; PMCID: PMC6433670.

75. Kamenova, I.; Mukherjee, P.; Conic, S.; Mueller, F.; El-Saafin, F.; Bardot, P.; Garnier, J.M.; Dembele, D.; Capponi, S.; Timmers, H.T.M. et al. Co-translational assembly of mammalian nuclear multisubunit complexes. Nat Commun. 2019; 10(1):1740. doi: 10.1038/s41467-019-09749-y. PMID: 30988355; PMCID: PMC6465333.

76. Grierson, P.M.; Lillard, K.; Behbehani, G.K.; Combs, K.A.; Bhattacharyya, S.; Acharya, S.; Groden, J. BLM helicase facilitates RNA polymerase I-mediated ribosomal RNA transcription. Hum Mol Genet. 2012; 21(5):1172-83. doi: 10.1093/hmg/ddr545. PMID: 22106380; PMCID: PMC3277314.

77. Xu, J.; Liu, Y.; Li, Y.; Wang, H.; Stewart, S.; Van der Jeught, K.; Agarwal, P.; Zhang, Y.; Liu, S.; Zhao, G. et al. Precise targeting of POLR2A as a therapeutic strategy for human triple negative breast cancer. Nat Nanotechnol. 2019; 14(4):388-397. doi: 10.1038/s41565-019-0381-6. PMID: 30804480; PMCID: PMC6449187.

78. Finlay-Schultz, J.; Gillen, A.E.; Brechbuhl, H.M.; Ivie, J.J.; Matthews, S.B.; Jacobsen, B.M.; Bentley, D.L.; Kabos, P.; Sartorius, C.A. Breast Cancer Suppression by Progesterone Receptors Is Mediated by Their Modulation of Estrogen Receptors and RNA Polymerase III. Cancer Res. 2017; 77(18):4934-4946. doi: 10.1158/0008-5472.CAN-16-3541PMID: 28729413; PMCID: PMC5600857.

79. Deng, J.; Hou, G.; Fang, Z.; Liu, J.; Lv, X.D. Distinct expression and prognostic value of OTU domaincontaining proteins in non-small-cell lung cancer. Oncol Lett. 2019; 18(5):5417-5427. doi: 10.3892/ol.2019.10883. PMID: 31612050; PMCID: PMC6781715. 
80. Cronin, N.B.; Yang, J.; Zhang, Z.; Kulkarni, K.; Chang, L.; Yamano, H.; Barford, D. Atomic-Resolution Structures of the APC/C Subunits Apc4 and the Apc5 N-Terminal Domain. J Mol Biol. 2015; 427(20):33003315. doi: 10.1016/j.jmb.2015.08.023. PMID: 26343760; PMCID: PMC4590430.

81. Agilent-026652 Whole Human Genome Microarray $4 \times 44 \mathrm{~K}$ v2. Available on line: https://www.ncbi.nlm.nih.gov/geo/query/acc.cgi?acc=GPL13497.

82. Choueiri, T.K.; Motzer, R.J. Systemic Therapy for Metastatic Renal-Cell Carcinoma. N Engl J Med. 2017; 376(4):354-366. doi: 10.1056/NEJMra1601333. PMID: 28121507.

83. Zbar, B.; Klausner, R.; Linehan, W.M. Studying cancer families to identify kidney cancer genes. Annu Rev Med. 2003; 54:217-33. doi: 10.1146/annurev.med.54.101601.152514. PMID: 12525673.

84. Di Nunno, V.; Mollica, V.; Brunelli, M.; Gatto, L.; Schiavina, R.; Fiorentino, M.; Santoni, M.; Montironi, R.; Caliò, A.; Eccher, A.; et al. A Meta-Analysis Evaluating Clinical Outcomes of Patients with Renal Cell Carcinoma Harboring Chromosome 9P Loss. Mol Diagn Ther. 2019; 23(5):569-577. doi: 10.1007/s40291-01900414-0. PMID: 31332726.

85. Iacobas, S.; Iacobas, D.A. Astrocyte proximity modulates the myelination gene fabric of oligodendrocytes. Neuron Glia Biol. 2010, 6, 157-169, doi:10.1017/S1740925X10000220.

Author Contributions: Conceptualization, D.A.I., H.W. and P.B.; methodology, D.A.I. and S.I.; software, D.A.I.; validation, D.A.I. and S.I.; formal analysis, D.A.I.; investigation, S.I.; resources, P.B.S.; data curation, K.M.M. V.E.M.; writing - original draft preparation, D.A.I.; writing - review and editing, P.B.S., H.S., K.M.M., V.E.M..; visualization, S.I..; supervision, D.A.I..; project administration, P.B.S.; funding acquisition, P.B.S. All authors have read and agreed to the published version of the manuscript.

Funding: This research was supported by the Texas A\&M University System Chancellor's Research Initiative (CRI) funding for the Radiation Institute for Science and Engineering (RaISE) and the Center for Computational Systems Biology (CCSB) at the Prairie View A\&M University.

Acknowledgments: The authors acknowledge the fruitful discussions with Dr. Abburi A. Kumar (PVAMU Electrical and Computer Engineering), Dr. Gloria Regisford (PVAMU - Biology), and Dr. Leah Bush (PVAMU Chemistry)

Conflicts of Interest: The authors declare no conflict of interest. 